\title{
Mass Hierarchy, Mixing, CP-Violation and Higgs Decay - or Why Rotation is Good for Us
}

\author{
Michael J BAKER』, José BORDES2, \\ CHAN Hong-Md? TSOU Sheung Tsunt
}

\begin{abstract}
The idea of a rank-one rotating mass matrix (R2M2) is reviewed detailing how it leads to ready explanations both for the fermion mass hierarchy and for the distinctive mixing patterns between up and down fermion states, which can be and have been tested against experiment and shown to be fully consistent with existing data. Further, R2M2 is seen to offer, as by-products: (i) a new solution of the strong $\mathrm{CP}$ problem in QCD by linking the theta-angle there to the KobayashiMaskawa CP-violating phase in the CKM matrix, and (ii) some novel predictions of possible anomalies in Higgs decay observable in principle at the LHC. A special effort is made to answer some questions raised.
\end{abstract}

\footnotetext{
${ }^{1}$ bakerm@maths.ox.ac.uk; Mathematical Institute, University of Oxford, 24-29 St. Giles', Oxford, OX1 3LB, United Kingdom; work supported by an EPSRC Research Studentship.

2jose.m.bordes@uv.es; Departament Fisica Teorica and IFIC, Centro Mixto CSIC, Universidad de Valencia, calle Dr. Moliner 50, E-46100 Burjassot (Valencia), Spain; work supported by Spanish MICINN under contract FPA2008-02878.

${ }^{3}$ hong-mo.chan @ stfc.ac.uk; Rutherford Appleton Laboratory, Chilton, Didcot, Oxon, OX11 OQX, United Kingdom.

${ }^{4}$ tsou @ maths.ox.ac.uk; Mathematical Institute, University of Oxford, 24-29 St. Giles', Oxford, OX1 3LB, United Kingdom.
} 


\section{Old Story}

Although almost all the material in this first half is published sometime ago, it is widely scattered in both time and space and in parts difficult of access. It is thus thought that collecting it now together and presenting it anew with the clarity acquired from experience and hindsight would help the reader judge better its significance. It would help also for evaluating better the more recent results to be reviewed in the second half, and offers at the same time an opportunity to address in detail some questions which might arise.

\subsection{Why R2M2?}

The need to inject from experiment the masses and mixing angles as inputs into the standard model subtracts much from our confidence in it as a fundamental theory in spite of all its phenomenological successes. These not only account for some two-thirds of its many empirical parameters, but are themselves a little mysterious, showing a hierarchical pattern begging explanation which is not supplied, even qualitatively, in the standard model framework. Presumably, the explanation will have to come elsewhere, perhaps in a more fundamental theory from which the standard model itself can be deduced as a consequence, and there is no lack of effort from the physics community to construct such a theory. However, before we succeed in doing so, it would be interesting to ask what sort of mechanism could give rise to such a hierarchical pattern so as to serve as a guideline for constructing our future theory. The mechanism will have to be somewhat unusual, given that the fermions of different generations are seen in experiment to have almost identical interactions, pointing to a hidden symmetry among them, which is only mildly broken, if at all. And yet the masses and mixing angles vary wildly from generation to generation, often by orders of magnitude. Any perturbative breaking of the generation symmetry is unlikely to give such a mass and mixing pattern. The rank-one rotating mass matrix (R2M2) is a suggestion for filling the gap.

The mass matrix obtained from the Yukawa term by substituting for the Higgs field its vacuum expectation value appears usually as:

$$
m \frac{1}{2}\left(1+\gamma_{5}\right)+m^{\dagger} \frac{1}{2}\left(1-\gamma_{5}\right)
$$

but by a suitable relabelling of the $s u(2)$-singlet right-handed fermion fields, which cannot change the physics, one can recast it in a hermitian form in which no $\gamma_{5}$ appears [1. Explicitly, any $m$ can always be diagonalized by 
unitary matrices $U$ and $V$, thus $U m V^{-1}=$ diagonal matrix. Then relabelling the right-handed fields $\psi_{R}$ as $U^{-1} V \psi_{R}$ will give a new mass matrix with the desired property. We shall always, in what follows, work with the mass matrix in this representation, in which case a rank-one mass matrix will appear simply as just a product of the eigenvector $\boldsymbol{\alpha}$ with the single nonzero eigenvalue times its hermitian conjugate, thus:

$$
m=m_{T} \boldsymbol{\alpha} \boldsymbol{\alpha}^{\dagger} .
$$

That one should perhaps start with a rank-one fermion mass matrix, i.e. one reducible to the form (2), where $\boldsymbol{\alpha}$, a unit vector in 3-D generation space, is the same for both the up and down states and only the coefficient $m_{T}$ depends on flavour (as in $m_{U}$ and $m_{D}$ ), has been suggested already some thirty years ago [2, 3]. This has only one massive generation and has the unit matrix as the mixing matrix, which is already not a bad first approximation, indeed tantalisingly close, to the experimental situation, at least for quarks. The idea then is that the small lower generation masses and deviations of the mixing matrix from the identity will arise from some perturbation to the above initial situation.

The difficult question, of course, is what perturbative mechanism will give rise to the hierarchical pattern of deviations from this first approximation. For example, in order to obtain non-trivial mixing between the up and down states, it would appear necessary at first sight to break the degeneracy of $\boldsymbol{\alpha}$ in up-down flavour. But the only forces we know in nature which depend on flavour come from the electroweak sector, and these seem too weak to give the desired effect; strong interactions, on the other hand, where one perhaps expects effects of such magnitudes to originate, are however flavour-blind.

That the mass matrix, or in the case of (2) the vector $\boldsymbol{\alpha}$, should rotate is a solution suggested to resolve this dilemma. By a rotating mass matrix, whether of rank one or not, we mean one the orientation of which in generation space depends on the renormalization scale. In other words, its eigenvectors change with scale. We are familiar already with mass matrices having eigenvalues which depend on scale, and hence give scale-dependent masses to the individual fermions [4], e.g. to the $b$-quark, which has even been tested experimentally. So a rotating mass matrix is just a further extension of the same idea of scale-dependence. The beauty of a rotating mass matrix is that even without breaking the degeneracy of $\boldsymbol{\alpha}$ in flavour, mixing and mass hierarchy will automatically emerge solely by virtue of the dependence of $\boldsymbol{\alpha}$ on scale. This means that mixing of sufficient magnitude can now in principle arise via rotation from strong interaction, which does not 
distinguish between up and down flavours. Of course, actually how it can come about there is itself an interesting question which will be outlined only briefly in a later subsection, since it is one of some intricacy and its resolution model-dependent, so that the reader will have to be referred elsewhere for details. Here, in this paper, R2M2 will be treated just as a phenomenological hypothesis to be tested against experiment and, if proved successful, to serve as hints for the construction of future theories to bolster, or perhaps eventually to supplant, the present standard model.

Now, for a rotating mass matrix, not only the masses but also the state vectors will depend on scale, which is a situation that one is not so familiar with and needs therefore more careful handling, giving results which may appear at first sight surprising. One will find indeed that there are certain conceptions one takes for granted because of familiarity which will have to be given up and replaced. One immediate example is precisely that it will give rise to the hierarchical mass and mixing patterns one seeks.

That a rank-one rotating mass matrix can give rise to mixing and mass hierarchy is a very simple idea which is most easily seen in the simplified situation when there are only two generations instead of the three in reality, where one takes account only of the two heaviest states in each fermion type. By (2) then, taking for the moment $\boldsymbol{\alpha}$ to be real and $m_{T}$ to be $\mu$-independent for simplicity, we would have $m_{t}=m_{U}$ as the mass of $t$ and the eigenvector $\boldsymbol{\alpha}\left(\mu=m_{t}\right)$ as its state vector $\mathbf{v}_{t}$. Similarly, we have $m_{b}=m_{D}$ as the mass and $\boldsymbol{\alpha}\left(\mu=m_{b}\right)$ as the state vector $\mathbf{v}_{b}$ of $b$. Next, the state vector $\mathbf{v}_{c}$ of $c$ must be orthogonal to $\mathbf{v}_{t}, c$ being by definition an independent quantum state to $t$. Similarly, the state vector $\mathbf{v}_{s}$ of $s$ is orthogonal to $\mathbf{v}_{b}$. So we have the situation as illustrated in Figure 1, where the vectors $\mathbf{v}_{t}$ and $\mathbf{v}_{b}$ are not aligned, being the vector $\boldsymbol{\alpha}(\mu)$ taken at two different values of its argument $\mu$, and $\boldsymbol{\alpha}$ by assumption rotates. This gives then the following CKM mixing (sub)matrix in the situation considered with only the two heaviest states:

$$
\left(\begin{array}{cc}
V_{c s} & V_{c b} \\
V_{t s} & V_{t b}
\end{array}\right)=\left(\begin{array}{cc}
\left\langle\mathbf{v}_{c} \mid \mathbf{v}_{s}\right\rangle & \left\langle\mathbf{v}_{c} \mid \mathbf{v}_{b}\right\rangle \\
\left\langle\mathbf{v}_{t} \mid \mathbf{v}_{s}\right\rangle & \left\langle\mathbf{v}_{t} \mid \mathbf{v}_{b}\right\rangle
\end{array}\right)=\left(\begin{array}{cc}
\cos \theta_{t b} & -\sin \theta_{t b} \\
\sin \theta_{t b} & \cos \theta_{t b}
\end{array}\right),
$$

which is no longer the identity: hence mixing.

Next, what about hierarchical masses? From (2), it follows that $\mathbf{v}_{c}$ must have zero eigenvalue at $\mu=m_{t}$. But this value is not to be taken as the mass of $c$ which has to be measured at $\mu=m_{c}$. In other words, $m_{c}$ is instead to be taken as the solution to the equation:

$$
\mu=\left\langle\mathbf{v}_{c}|m(\mu)| \mathbf{v}_{c}\right\rangle=m_{U}\left|\left\langle\mathbf{v}_{c} \mid \boldsymbol{\alpha}(\mu)\right\rangle\right|^{2} .
$$




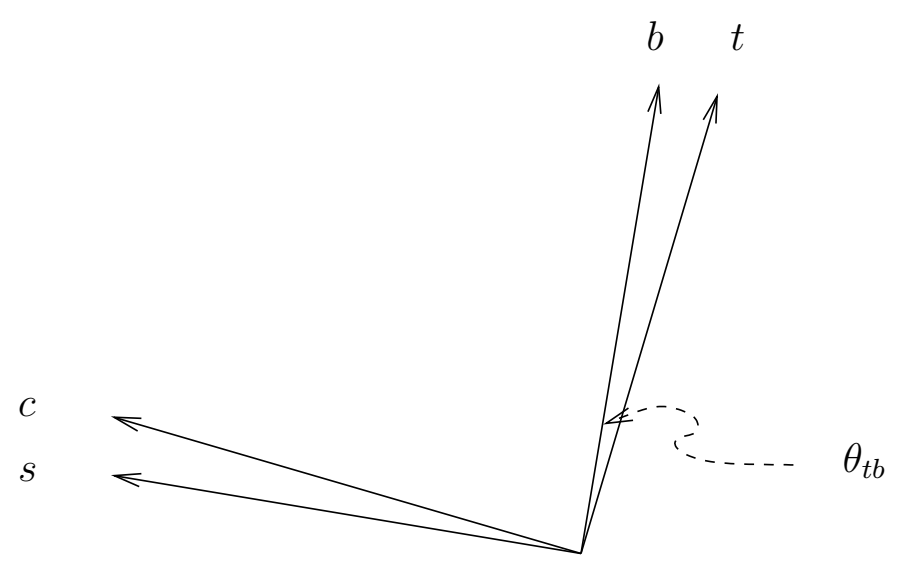

Figure 1: Illustration of $U D$ mixing for two generations.

A nonzero solution exists since the scale on the LHS decreases from $\mu=m_{t}$ while the RHS increases from zero at that scale. Another way to see this is that since $\boldsymbol{\alpha}$ by assumption rotates so that at $\mu<m_{t}$, it would have rotated to some direction different from $\mathbf{v}_{t}$, as illustrated in Figure 2, and acquired a component, say $\sin \theta_{t c}$, in the direction of $\mathbf{v}_{c}$ giving thus:

$$
m_{c}=m_{t} \sin ^{2} \theta_{t c}
$$

This is nonzero but will be small if the rotation is not too fast: hence mass hierarchy.

We see then that with 2 generations, rotation will give both mixing and mass hierarchy. Basically the same argument can be applied also to the realistic 3-generation case, but the situation becomes more intricate and therefore more interesting, deserving a separate and more detailed treatment later. Before we do so, however, let us first ask a few urgent questions triggered by the assertions above.

\subsection{Does rotation work?}

The first is obviously the following. Granted that R2M2 does give rise to mass hierarchy and mixing, are the mass spectrum and mixing pattern anywhere near what is experimentally observed? In the planar approximation discussed in the preceding subsection, this question can easily be answered. As seen in (3) and (5) the mixing angles and mass ratios are given explicitly in terms of the rotation angle. Hence, given the experimentally measured values of 


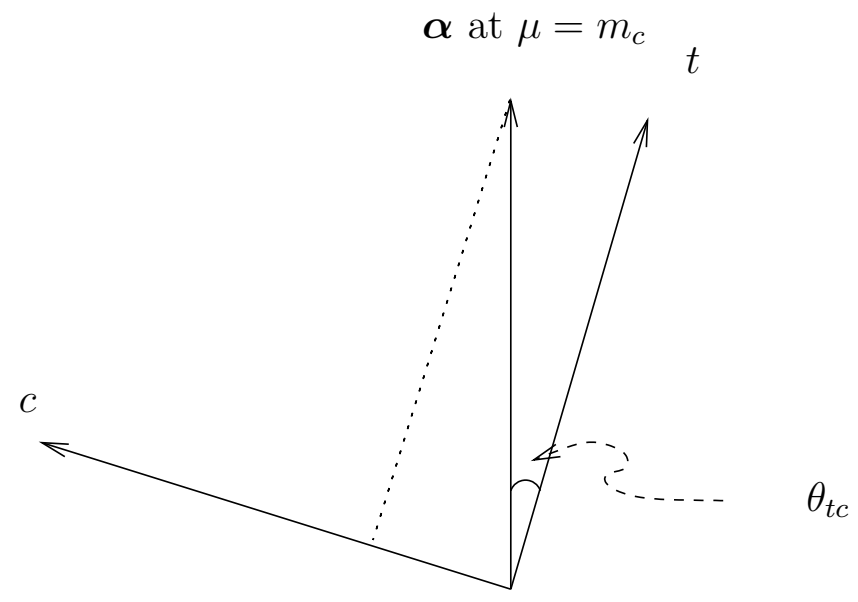

Figure 2: Illustration of mass leakage for two generations.

these quantities, one can easily invert the formulae to evaluate the values of the rotation angle to which they correspond. Then, if R2M2 works, the rotation angles so obtained when plotted against the scales at which the various mixing angles and masses are measured should all lie on a smooth curve representing the rotation trajectory of the vector $\boldsymbol{\alpha}$ appearing in the mass matrix (2). This exercise was done in [5] and is reproduced here in Figure 3. This clearly shows that the data then available were perfectly consistent with the R2M2 hypothesis in the "planar approximation" in which account is taken only of the 2 heaviest generations.

Whether the rotation mechanism will still work in the realistic situation when all 3 fermion generations are taken into account will be considered later when the 3-G analysis has been performed. One can ask at this stage, however, why is it that by missing out the lowest generation as was done above, one still obtains a sensible picture. For 3 generations $(3-G)$, the vector $\boldsymbol{\alpha}$ will trace out for changing $\mu$ a curve on the unit sphere. By restricting oneself to only the two heaviest generations, one is projecting this space curve on to the plane containing the state vectors of the two heaviest generations, and this "planar approximation" would be reasonable so long as the curve in the region studied is nearly planar. As will be seen later, the degree of nonplanarity of the trajectory for $\boldsymbol{\alpha}$ in the region considered is measured by the Cabibbo angle $\sim 0.22$, and so long as one stays in that region, nonplanar effects are only of the order $0.22^{2} \sim 0.04$ and are negligible to the accuracy needed [5]. Beyond the region shown in Figure [3, i.e. for, say, $\mu \leq m_{\mu}$, 


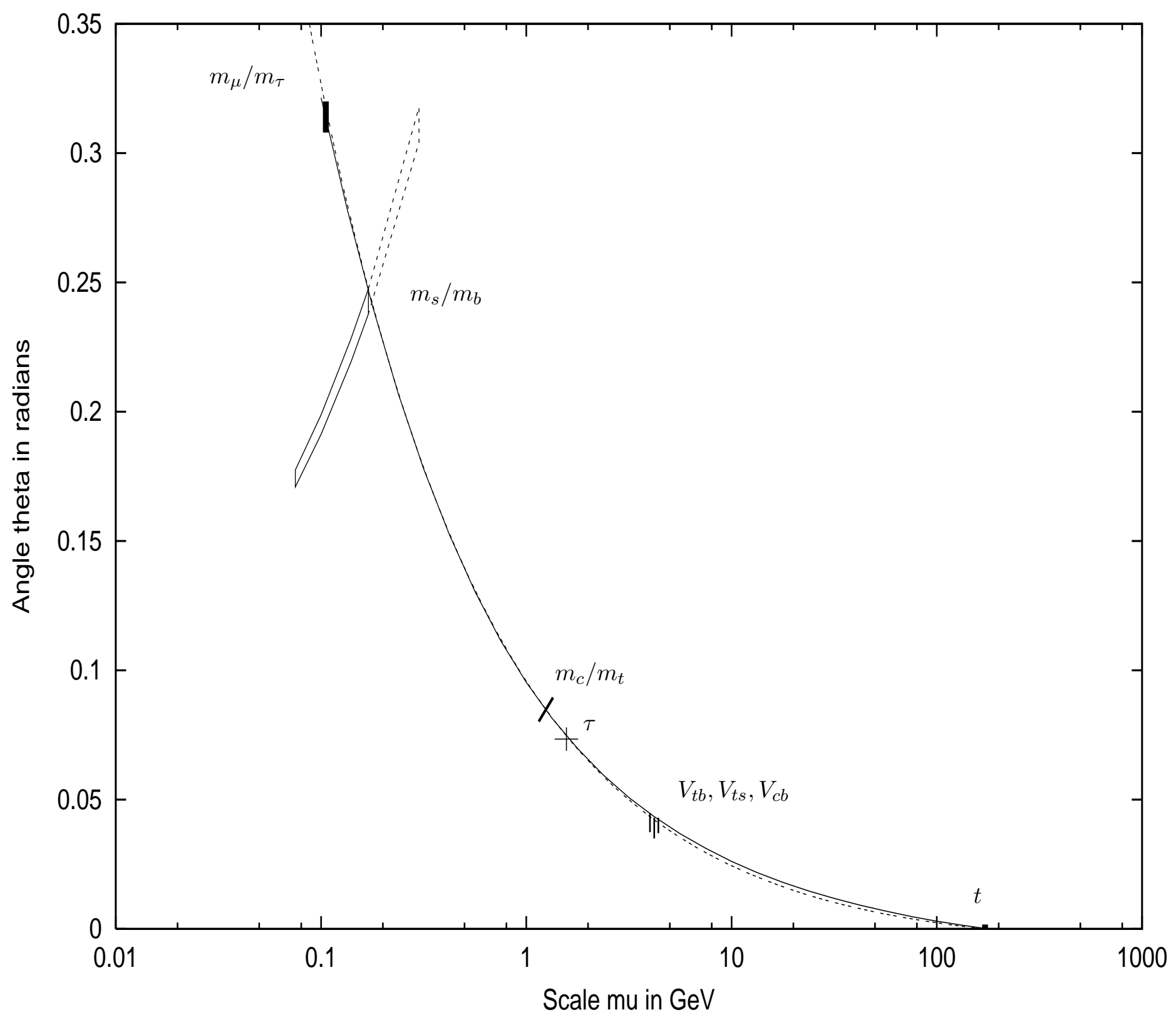

Figure 3: The rotation angle changing with scale as extracted from the data on mass ratios and mixing angles, in planar approximation, taken from [5]. 
nonplanar effects will have to be taken into account, as will be done when the time comes.

The exercise summarized in Figure 3, apart from answering the question posed above, also gives some additional indications for rotation which were at first not bargained for.

(U) In first proposing the formula (2) for the rotating mass matrix, one is guided by the experimental fact that the CKM mixing matrix [6, 17] is close to unity to insert the same vector $\boldsymbol{\alpha}$ for the up and down quark states, as suggested a long time ago already [2, 3]. But there is a priori no similar reason to suggest that the mass matrix for charged leptons should also have the same $\boldsymbol{\alpha}$. Nevertheless, one finds in Figure 3 that the masses for the charged leptons $\tau$ and $\mu$ actually fit very well on to the same trajectory, pointing to some sort of universality for $\boldsymbol{\alpha}$.

(F) As seen in Figure 3, the trajectory for $\boldsymbol{\alpha}$ seems to approach an asymptote as $\mu \rightarrow \infty$. Indeed, the best empirical fit to the data points as shown in the figure is an exponential, indicative of a rotational fixed point at $\mu=\infty$, meaning that the rotation will get progressively slower for larger $\mu$. This can be seen also from the experimental values of the mass ratios: $m_{\mu} / m_{\tau}>m_{s} / m_{b}>m_{c} / m_{t}$ getting progressively smaller as the scale increases, since in the rotation scenario, it is the leakage by rotation which gives rise to the mass ratio of the second to the heaviest generations, and as the rotation rate gets smaller, so will the leakage.

These two observations will be designated in what follows as assumptions $(\mathbf{U})$ and $(\mathbf{F})$. Though initially quite independent of the R2M2 hypothesis, they will enhance by much its predictive power, and may be taken as additional hints for constructing the future theory behind the standard model.

\subsection{How does rotation arise?}

The R2M2 hypothesis has two components, namely that the fermion mass matrix is first, of rank one, and secondly, rotating with changing scale. The two components are not contingent one on the other, for clearly the mass matrix can be of rank one without rotating, or it can rotate without being of rank one. Although the two components conspire in the application to give the explanation for mixing and mass hierarchy detailed above, in theoretical considerations, it is more convenient to separate the two so as to clarify the different issues involved. 
That it seems expedient to start with a mass matrix of rank one is an old idea [2, 3] with which one would be familiar with, but that it should rotate is a proposal not often met with in the literature except in the present context, and it would be appropriate, before we go any further, to ask first why, and under what conditions, would a mass matrix rotate.

The answer is easy to find, for even in the standard model as usually formulated, so long as there is nontrivial mixing between up and down fermion states, then rotation of the fermion mass matrices will occur. This was pointed out already long ago in e.g. [8], although not often noted, and can easily be seen as follows. The renormalization group equation for the mass matrices $U$ and $D$ of respectively the up and down quarks to leading order can be written as $[9]$ :

$$
\begin{aligned}
16 \pi^{2} \frac{d U}{d t} & =\frac{3}{2}\left(U U^{\dagger}-D D^{\dagger}\right) U+\left(\Sigma_{U}-A_{U}\right) U \\
16 \pi^{2} \frac{d D}{d t} & =\frac{3}{2}\left(D D^{\dagger}-U U^{\dagger}\right) D+\left(\Sigma_{D}-A_{D}\right) D,
\end{aligned}
$$

where in each equation the first term on the right represents the contribution of the Higgs boson loop while the second term that of the gluon loop. Of the two terms, what is of interest here is the former, where we recall that by nontrivial mixing between up and down states, we mean that the matrices $U$ and $D$ are related by a nondiagonal matrix $V$. Thus $D=V U V^{\dagger}$ or $U=$ $V^{\dagger} D V$, so that $U$ and $D$ cannot be simultaneously diagonalized. Suppose now at some $t=\ln \mu$ we diagonalize $U$ in (6) by a unitary transformation, which we can since $U$ is hermitian. All terms in (6) are then diagonal except $D D^{\dagger} U$, which will thus necessarily de-diagonalize the matrix $U$ on running by (6) to a different $t$.

In other words the matrix $U$ will rotate with changing $t$ or $\mu$, as asserted. Similar arguments hold also for the rotation of the matrix $D$. They will apply as well to the lepton mass matrices given that experiments on neutrino oscillation imply a nontrivial (PMNS) mixing [10] also for leptons. The only reason why this fact is not more commonly noted is that the effect is small, given the smallness of the up-down mixing itself which is driving the rotation.

We conclude therefore that there is actually nothing very unusual in the stipulation in R2M2 of a mass matrix which rotates with changing scale, and that rotation is connected to mixing, since it occurs already in the standard model as commonly formulated. What is unusual is only that instead of the up-down mixing giving rise to the rotation as demonstrated above for the standard model, one wants instead that it is the rotation of the fermion mass 
matrix which is giving rise to the up-down mixing. This means two things: first, that the rotation has to be much faster than is generated by the above equations (6) and (7), and secondly, that it has to be driven by some other effects yet to be identified which lie outside the standard model framework.

Clearly, if that is the direction one wants to go, what is needed is some new renormalization effect which will give a term in the renormalization group equation that is not diagonal when the mass matrix is diagonal. This looks like that one will have to look for some more fundamental theory which will give rise to such an effect and is at the same time compatible with the standard model so as not to destroy its near-perfect agreement with experiment at present. And one will, presumably, want this theory also to have a reasonably appealing theoretical foundation.

An attempt along these lines [11, 12, 13], suggested by ourselves and designated as the framed standard model (FSM), will be reported elsewhere [14]. This starts from the premises of what could be called a framed gauge theory (FGT) framework [15] where in addition to the usual gauge boson and matter fermion fields of ordinary gauge theories, one introduces also the frame vectors (vielbeins) in internal symmetry space as dynamical variables (framons), as one does the vierbeins in gravity. Just as the vierbeins in gravity, the framons in FGT will carry two types of indices, referring one to the local, and the other to the global, reference frames, and the action will have to be invariant under both the local and global symmetries. The FSM appears in this FGT framework as the "minimally framed" theory with the standard model gauge symmetry $s u(3) \times s u(2) \times u(1)$, by virtue of which fact, it has been shown to lead automatically to the following results:

- It contains within its structure a scalar boson to play the role of the usual Higgs field, as well as a global su(3) symmetry to play the role of fermion generations.

- It gives a fermion mass matrix of the factorizable form (21) with $\boldsymbol{\alpha}$ independent of the fermion type, i.e. satisfying assumption (U) of $\S 1.2$ above.

- By virtue of the required invariance under both the local and global symmetries, the vacuum is degenerate and is coupled to the vector $\boldsymbol{\alpha}$ appearing in fermion mass matrix (2).

- Under renormalization in the strong sector, the vacuum is found to change with scale, and drags $\boldsymbol{\alpha}$ along with it; hence rotation. 
- This rotating $\boldsymbol{\alpha}$ has a fixed point at infinite scale, i.e. satisfying assumption $(\mathbf{F})$ of $\S 1.2$ above.

In other words, the FSM satisfies R2M2 and possesses the properties identified above as essential for R2M2 to reproduce fermion mixing and mass hierarchy, while keeping still the main features of the standard model. At the same time, it is based on premises with, we think, a fair amount of theoretical appeal.

However, for the purpose of the present paper, the FSM serves only as an example that such rotational models are possible, while R2M2 itself will continue to be treated as a phenomenological hypothesis. In other words considerations are given only to those consequences of R2M2 which are independent of what physical mechanism is driving the rotation, so as the better to serve as a guide for model builders who may be starting with very different ideas.

\subsection{Stumbling block?}

At this juncture two questions, basically the same, may be asked, and indeed are often asked, which need to be addressed before one proceeds any further. Since they can be both posed and clarified already in the planar approximation of $\S 1.1$ with only 2 generations, it will now be done below.

Q1. The mass matrix of (2) at any $\mu$ is invariant under a chiral transformation on the state orthogonal to $\boldsymbol{\alpha}(\mu)$, in particular at $\mu=m_{t}$, under a chiral transformation in the state $\mathbf{v}_{c}$. Chiral invariance is associated with zero mass particles. Yet, it was claimed in $\S 1.1$ that $m_{c}$ is nonzero. Why?

Q2. The mass matrix $m$ in (2) has one zero eigenvalue at every $\mu$, in particular at $\mu=m_{t}$, this zero eigenvalue has as eigenvector $\mathbf{v}_{c}$. The eigenvalues of a mass matrix are by definition the masses of particles. Yet it was claimed that $m_{c}$ is nonzero. Why?

The point raised in these questions, though accentuated by the fact that the mass matrix is of rank one, concerns mainly only the other component of the R2M2 hypothesis, namely that the mass matrix rotates with changing scale. Let us therefore start here with a rotating mass matrix, not necessarily of rank one so as not to confuse the issue, only returning at the end to the rank-one case to answer the questions posed. 
Now, the common concepts concerning the mass matrix, such as those set out in italics in Q1 and Q2 above, were obtained at first on the premises where $m$ has no scale-dependence. On extending the consideration to scaledependent and then to rotating mass matrices, one ought therefore logically to re-examine whether the concepts we have gathered before still remain valid. This is particularly true for the rotating mass matrix, which is, in a sense, uncharted waters needing some caution to negotiate, for although it occurs already in the standard model as noted in the preceding subsection, the effect there was small and has not engaged much attention. It is thus proposed that one goes back to the beginning where the idea of a mass matrix was first conceived, then work back step by step to the presently desired rotating, rank-one case. Notice that such an analysis would in any case be needed theoretically, even if one is satisfied with the standard model as it stands, and eventually even needed practically when experimental accuracy reaches a point where the previously ignored effects of rotation become measurable. Only the search now for an explanation of mixing and mass hierarchy via rotation has brought the necessity forward.

As already noted, the mass matrix when originally conceived was a scaleindependent quantity, being the value of the Yukawa term when the Higgs field appearing in it is given its vacuum expectation value. Then indeed, its eigenvalues are to be interpreted as the masses of the physical particles. After that one learns that the mass matrix so defined actually depends on the renormalization scale. One meets first with the situation when only the eigenvalues $m_{1}(\mu)$ and $m_{2}(\mu)$ of the mass matrix are scale-dependent, not its respective eigenvectors $\mathbf{v}_{1}$ and $\mathbf{v}_{2}$, i.e. the non-rotating case, which is a fair approximation to the standard model as aleady noted. Once the quantities involved are dependent on the scale, one would have to specify at what scales physical parameters are to be measured. The standard convention is that the mass of each individual particle is to be measured at the scale equal to its mass. Thus, specializing for the moment to the $U$-type quarks for the case under consideration, when only the eigenvalues depend on scale, the mass of the $t$-quark is the scale at which the larger eigenvalue, say $m_{1}(\mu)$, is equal to the scale itself, i.e. the solution to the equation:

$$
m_{1}(\mu)=\mu
$$

in other words, we find both the $t$-mass and its state vector:

$$
m_{1}\left(m_{t}\right)=m_{t} \quad \text { and } \quad \mathbf{v}_{1}=\mathbf{v}_{t}
$$


By orthogonality,

$$
\mathbf{v}_{2}=\mathbf{v}_{c}
$$

Similarly, the mass of the $c$-quark is defined as the scale at which the smaller of the two eigenvalue say $m_{2}(\mu)$ is equal in value to the scale itself, i.e. solution to the equation (8), only with 2 instead of 1 , so that we have

$$
m_{2}\left(m_{c}\right)=m_{c},
$$

which is not equal to $m_{2}\left(m_{t}\right)$. This is by now familiar. Notice, however, that at this stage we have already departed from the original assertion set out in italics in Q2 above that the physical masses of particles are the eigenvalues of "the" mass matrix. The two physical masses of $t$ and $c$ are both indeed eigenvalues, but they are evaluated at different scales, meaning that they are two eigenvalues of two distinct matrices $m\left(\mu=m_{t}\right)$ and $m\left(\mu=m_{c}\right)$. There is in fact no single matrix among the set $m(\mu)$ which has $m_{t}$ and $m_{c}$ as its two eigenvalues. In other words, the designation of $m(\mu)$ as the mass matrix has become only nominal, for in no case are the eigenvalues of a single $m(\mu)$ at any given $\mu$ the physical masses of the particles $t$ and $c$.

Indeed, there is yet another sense in which the matrix $m(\mu)$ has departed from the idea of a mass matrix as originally conceived. At any energy scale $m_{c}<\mu<m_{t}$, the state $t$ cannot appear as a physical state, and only the state $c$ remains physical. So there should not be a mass matrix at all but just a number representing the scale-dependent "mass" of the $c$-quark, with no matrix elements referring to the $t$ state at all. Recall the analogous case of the analytic two-channel $S$-matrix [16], say,

$$
S=\left(\begin{array}{cc}
S_{a a} & S_{a b} \\
S_{b a} & S_{b b}
\end{array}\right) .
$$

It is a $2 \times 2$ matrix and exists for all energies $E$, but for $E_{b}<E<E_{a}$, where $E_{a}$ and $E_{b}$ denote respectively the energy thresholds for the channels $a$ and $b$, it is only nominally the $S$-matrix, because in that energy range, the channel $a$ becomes unphysical. The physical $S$-matrix, which by unitarity should refer only to physical channels, is there given just by the element $S_{b b}$, obtained via a truncation of the matrix (12) by removing the rows and columns referring to the unphysical channel $a$. It would thus seem that the same should hold for the scale-dependent mass matrix $m(\mu)$ under consideration. For $m_{c}<\mu<$ $m_{t}$, the matrix $m(\mu)$ is only nominally the mass matrix. The physical mass matrix is obtained by truncating $m(\mu)$ by removing the rows and columns referring to the state $t$ leaving only a number referring to the space orthogonal 
to $\mathbf{v}_{t}$, i.e. in the direction of $\mathbf{v}_{c}$. That is, it ought to be the case in principle, but in practice, one sees that in the case being considered it does not really matter. When the matrix $m(\mu)$ is diagonalized at any $\mu$ in the non-rotating case we are at present considering, it will remain diagonalized for all $\mu$. Then truncating $m(\mu)$ as prescribed above will leave us just the number $m_{2}(\mu)$. So the mass of $c$ is still to be obtained by solving the equation $m_{2}(\mu)=\mu$ as was suggested, a result we have now confirmed by a more careful analysis.

The interesting question now is what happens when this "nominal" mass matrix $m(\mu)$ changes not only its eigenvalues $m_{1}(\mu)$ and $m_{2}(\mu)$, but its eigenvectors $\mathbf{v}_{1}(\mu)$ and $\mathbf{v}_{2}(\mu)$ as well, as the scale changes. In other words, what happens if $m(\mu)$ rotates? As before, the $t$ mass is the solution to equation (8)), giving both the mass and state vector of $t$ :

$$
m_{1}\left(m_{t}\right)=m_{t} \quad \text { and } \quad \mathbf{v}_{1}\left(m_{t}\right)=\mathbf{v}_{t} .
$$

By orthogonality,

$$
\mathbf{v}_{2}\left(m_{t}\right)=\mathbf{v}_{c} .
$$

In contrast to the nonrotating case, however, the truncation required by unitarity for scales $\mu<m_{t}$ is no longer quite so uneventful as before. For $\mu<m_{t}$, what has to be projected out is the state $t$ which becomes unphysical. What remains as the physical mass matrix for $m_{c}<\mu<m_{t}$ is the submatrix in the subspace orthogonal to $\mathbf{v}_{t}$,

$$
\hat{m}(\mu)=\left\langle\mathbf{v}_{c}|m(\mu)| \mathbf{v}_{c}\right\rangle
$$

which is just a number in this simple 2-generation model, The physical mass of $c$ is the value of the scale $\mu$ when this number is equal to the scale itself, namely the solution to the first equation in (4) of $\S 1.1$ above. Explicitly,

$$
\hat{m}\left(m_{c}\right)=\left\langle\mathbf{v}_{c}\left|m\left(m_{c}\right)\right| \mathbf{v}_{c}\right\rangle=m_{c}
$$

which is not equal to

$$
m_{2}\left(m_{c}\right)=\left\langle\mathbf{v}_{2}\left(m_{c}\right)\left|m\left(m_{c}\right)\right| \mathbf{v}_{2}\left(m_{c}\right)\right\rangle
$$

since

$$
\mathbf{v}_{2}\left(m_{c}\right) \neq \mathbf{v}_{2}\left(m_{t}\right) \text { and } \quad \mathbf{v}_{c}=\mathbf{v}_{2}\left(m_{t}\right),
$$

because $\mathbf{v}_{2}\left(\mu=m_{c}\right)$ is necessarily orthogonal to $\mathbf{v}_{1}\left(\mu=m_{c}\right)$, but not, as $m(\mu)$ rotates, orthogonal to $\mathbf{v}_{t}=\mathbf{v}_{1}\left(\mu=m_{t}\right)$ as $\mathbf{v}_{c}$ is. We conclude therefore 
that the physical mass of $m_{c}$ of $c$ is not an eigenvalue of the "nominal" mass matrix $m(\mu)$ at $\mu=m_{c}$, nor indeed necessarily at any other value of $\mu$.

One may ask what if one ignores truncation and insists on taking the eigenvalue $m_{2}(\mu)$ at $\mu=m_{c}$ as the mass of the $c$ quark just as one did above for the case when $m(\mu)$ was not rotating. Then one would have the eigenvector $\mathbf{v}_{2}\left(\mu=m_{c}\right)$ as the state vector of $c$, which is orthogonal to the other eigenvector of $m(\mu)$ at $\mu=m_{c}$, namely $\mathbf{v}_{1}\left(\mu=m_{c}\right)$. But this means that it cannot be orthogonal to $\mathbf{v}_{t}$ which is $\mathbf{v}_{1}\left(\mu=m_{t}\right)$, and $\mathbf{v}_{1}\left(\mu=m_{t}\right) \neq$ $\mathbf{v}_{1}\left(\mu=m_{c}\right)$ since the mass matrix $m(\mu)$ by assumption rotates. In other words, the state vector $\mathbf{v}_{c}$ of $c$ would have a component in the direction of the state vector $\mathbf{v}_{t}$ of $t$, supposedly an independent quantum state to $c$, contradicting thus unitarity.

With this conclusion in hand, let us return to the questions Q1 and Q2 posed at the beginning of the subsection. Specializing now to a rotating mass matrix of rank one, namely (2), gives the value of $m_{c}$ via the second equation in (44) of $\S 1.1$, which we have already remarked is nonzero. But this is now seen to pose no problem since the conclusion is that physical masses of particles for a rotating scenario need not, and in general are not, eigenvalues of the rotating nominal mass matrix for non-leading generations. Hence $m_{c}$ need not be zero even when the nonleading eigenvalue is zero for $m(\mu)$ at all $\mu$. This thus falsifies the assertion in italics in Q2 and answers at the same time that question. It also answers the question Q1 since the chiral symmetry of the nominal mass matrix $m(\mu)$ appearing in the action will be maintained so long as this matrix has a zero eigenvalue, which it always has, but this is not contradicted by the physical $c$ mass $m_{c}$ being nonzero, since $m_{c}$ is not an eigenvalue of $m(\mu)$ at $\mu=m_{c}$ nor necessarily at any other value of $\mu$, falsifying thus the assertion in italics there.

\subsection{Three generations: the realistic case}

Having clarified in preceding subsections some immediate questions concerning the R2M2 hypothesis with the simplified 2-generation model, we are now ready to apply $\mathrm{R} 2 \mathrm{M} 2$ to the realistic 3-generation case.

For three generations of fermions, the vector $\boldsymbol{\alpha}$ in the rank-one mass matrix (2) is a 3 -vector, and as the scale $\mu$ varies, $\boldsymbol{\alpha}$ traces out a trajectory on the unit sphere. Now a space curve has of course more freedom than a plane curve, leading to more intricate features for both the mass spectrum and mixing pattern of fermions. While a plane curve can bend only one way, a space curve on a surface can bend with the surface (normal curvature $\kappa_{n}$ ) or 
sideways (geodesic curvature $\kappa_{g}$ ), and it can also twist (torsion $\tau$ ) [17], but if the surface is the unit sphere, as in our case, then the relevant twist, namely the geodesic torsion $\tau_{g}$, is zero. This means that whereas the amount of bending achieved by a curve in either direction, i.e. whether with the surface or sideways, over a small displacement in arc-length $\delta s$ is proportional to $\delta s$, namely $\kappa_{n} \delta s$ or $\kappa_{g} \delta s$, any twist achieved over the same displacement will have to be of at least second order in $\delta s$. As will be seen, this simple geometric fact will already go a long way towards explaining the hierarchical mass spectrum and mixing pattern of fermions observed in experiment.

Let us first recall here the experimental facts which we are meant to explain. According to the PDG data [18], the fermion mass spectrum is roughly as follows:

$$
\begin{array}{lll}
m_{t} \sim 171.3 \mathrm{GeV} & m_{b} \sim 4.20 \mathrm{GeV} & m_{\tau} \sim 1.777 \mathrm{GeV} \\
m_{c} \sim 1.27 \mathrm{GeV} & m_{s} \sim 105 \mathrm{MeV} & m_{\mu} \sim 106 \mathrm{MeV} \\
m_{u} \sim 2 \mathrm{MeV} & m_{d} \sim 5 \mathrm{MeV} & m_{e} \sim 0.5 \mathrm{MeV}
\end{array}
$$

and

$$
\left|\Delta m_{32}^{2}\right| \sim 2.43 \times 10^{-3} \mathrm{eV}^{2} ; \quad\left|\Delta m_{21}^{2}\right| \sim 7.59 \times 10^{-5} \mathrm{eV}^{2}
$$

while the CKM mixing matrix elements (absolute values) for quarks appear as:

$$
\left|V_{C K M}\right| \sim\left(\begin{array}{lll}
0.97419 & 0.2257 & 0.00359 \\
0.2256 & 0.97334 & 0.0415 \\
0.00874 & 0.0407 & 0.999133
\end{array}\right),
$$

and the PMNS mixing angles for leptons are given by:

$$
\sin ^{2} \theta_{12} \sim 0.32, \sin ^{2} \theta_{13}<0.05, \sin ^{2} \theta_{23}>0.36 \text {. }
$$

To explain the actual values of the mass and mixing parameters would be the aim of an eventual theory behind the standard model, but for the R2M2 hypothesis of our concern here the main interest is to first understand the qualitative patterns.

For these, in the mass spectrum, one can identify the following:

(A1) For each of the 3 fermion types $U, D$, and the charged leptons $L$, the masses decrease by a large factor, each of an order of magnitude or more, from generation to generation. For neutrinos, although it is not yet clear whether their masses follow the same hierarchical pattern, existing data are fully consistent with it. 
(A2) This factor, say between the second and the heaviest generations, changes from type to type, increasing with decreasing mass of the heaviest generation in the fermions type, thus: $m_{c} / m_{t}<m_{s} / m_{b}<m_{\mu} / m_{\tau}$, except again possibly for neutrinos.

(A3) The masses in each generation also seem to change "hierarchically" across the different types thus: $m_{t} \gg m_{b}>m_{\tau} \gg m_{\nu_{3}}$, but here it is not clear what decides the ordering. An exception is that $m_{u}<m_{d}$, bucking the trend, which is of course a crucial fact that guarantees the stability of the proton, and hence of us.

Whilst in the mixing matrices, one can identify the following:

(B1) The matrix elements for both quarks and leptons seem to decrease hierarchically away from the diagonal, with the elements at the corners (13 or 31) being at least about an order of magnitude smaller than the others $(12,23,21,32)$.

(B2) The element (23) for quarks is much smaller than that for leptons, while the element (12) remains about the same for both, hence giving $V_{u s}, V_{c d} \gg V_{c b}, V_{t s}$ for quarks.

We would like now to see whether the R2M2 hypothesis is able to explain these.

Let us examine first the mass hierarchy in a single fermion type, say the $U$-type quarks $t, c, u$. Extending the analysis for the $2-\mathrm{G}$ case above leading to the formula (4) for the $c$ mass to the present 3-G case, one obtains straightforwardly:

$$
\begin{aligned}
m_{t} & =m_{U}, \\
m_{c} & =m_{U}\left|\boldsymbol{\alpha}_{c} \cdot \mathbf{v}_{c}\right|^{2}, \\
m_{u} & =m_{U}\left|\boldsymbol{\alpha}_{u} \cdot \mathbf{v}_{u}\right|^{2} .
\end{aligned}
$$

where one has yet to specify what are the vectors $\mathbf{v}_{c}$ and $\mathbf{v}_{u}$. For the $2-G$ case, the assertion that the $c$ and $t$ are independent quantum states and hence should have orthogonal state vectors already specifies $\mathbf{v}_{c}$, given that $\mathbf{v}_{t}=\boldsymbol{\alpha}\left(m_{t}\right)$. Here in the 3-G case, however, the same assertion still leaves us the ambiguity of which vector on the plane orthogonal to $\mathbf{v}_{t}=\boldsymbol{\alpha}\left(m_{t}\right)$ is to be taken as $\mathbf{v}_{c}$, although, of course, once having decided on $\mathbf{v}_{c}$, then $\mathbf{v}_{u}$ is determined. 
This ambiguity is resolved as follows. For scales $\mu<m_{t}$, the state $t$ becomes unphysical, hence also the "mass matrix" $m$ in (2). According to the discussion in the preceding section, the mass matrix for these scales is to be obtained from the nominal matrix $m$ by projecting out the unphysical $t$-state into the subspace orthogonal to $\mathbf{v}_{t}$, which gives for the present case:

$$
\hat{m}_{i j}=m_{U}\left\langle\mathbf{v}_{i} \mid \boldsymbol{\alpha}\right\rangle\left\langle\boldsymbol{\alpha} \mid \mathbf{v}_{j}\right\rangle
$$

where $\mathbf{v}_{i}, i=1,2$ are any two basis vectors chosen on the said orthogonal subspace. Notice that this truncation leaves the $2 \times 2$ mass matrix $\hat{m}$ for $\mu<m_{t}$ still of the factorized form as was the original $3 \times 3$ matrix $m$. Explicitly:

$$
\hat{m}=\hat{m}_{U} \hat{\boldsymbol{\alpha}} \hat{\boldsymbol{\alpha}}^{\dagger}
$$

if we put:

$$
\hat{m}_{U}=m_{U} N
$$

with

$$
\begin{gathered}
N=\sqrt{\left|\left\langle\mathbf{v}_{1} \mid \boldsymbol{\alpha}\right\rangle\right|^{2}+\left|\left\langle\mathbf{v}_{2} \mid \boldsymbol{\alpha}\right\rangle\right|^{2}}, \\
\hat{\boldsymbol{\alpha}}=N^{-1}\left(\begin{array}{c}
\left\langle\mathbf{v}_{1} \mid \boldsymbol{\alpha}\right\rangle \\
\left\langle\mathbf{v}_{2} \mid \boldsymbol{\alpha}\right\rangle
\end{array}\right),
\end{gathered}
$$

and $\hat{\boldsymbol{\alpha}}$ as the 2-vector factorized from $\hat{m}$ in the subspace orthogonal to $\mathbf{v}_{t}$. In other words, the situation is entirely analogous to the $3 \times 3$ mass matrix that one started with originally, only now in one lower dimension. One concludes therefore that, as for $\mathbf{v}_{t}$ in $m$ before, the state vector $\mathbf{v}_{c}$ of $\mathrm{c}$ should likewise be taken as the unique massive eigenstate of $\hat{m}$, namely $\hat{\boldsymbol{\alpha}}(\mu)$, taken at scale $\mu=m_{c}$. The state vector $\mathbf{v}_{u}$ is then determined by orthogonality to both $\mathbf{v}_{t}$ and $\mathbf{v}_{c}$. Explicitly, we have then the whole $U$-triad as:

$$
\begin{aligned}
\mathbf{v}_{t} & =\boldsymbol{\alpha}\left(m_{t}\right) \\
\mathbf{v}_{c} & =\mathbf{v}_{u} \times \mathbf{v}_{t} ; \\
\mathbf{v}_{u} & =\frac{\boldsymbol{\alpha}\left(m_{t}\right) \times \boldsymbol{\alpha}\left(m_{c}\right)}{\left|\boldsymbol{\alpha}\left(m_{t}\right) \times \boldsymbol{\alpha}\left(m_{c}\right)\right|}
\end{aligned}
$$

Coupled with (23), these equations then give all the masses and state vectors of the $U$-type quarks.

That the mass spectrum of $U$-type quarks so obtained should be hierarchical, i.e. $m_{t} \gg m_{c} \gg m_{u}$, can now be seen from the earlier geometric observations as follows. From the definition of the $U$-triad in (29) above, one sees that the $c$ mass $m_{c}$ is associated with the bending (curvature) of the 
trajectory while the $u$ mass is associated with the twist (geodesic torsion). Then the fact cited before that the rotation trajectory on a round sphere has no geodesic torsion implies that while $\sqrt{m_{c}}$ is of first order in displacement along the trajectory, $\sqrt{m_{u}}$ is necessarily of second or higher order.

The same conclusion can be seen in perhaps more physical language. The state vector $\mathbf{v}_{c}$ of $c$ is the (normalized) vector orthogonal to $\mathbf{v}_{t}$ lying on the plane containing both $\boldsymbol{\alpha}\left(\mu=m_{t}\right)$ and $\boldsymbol{\alpha}\left(\mu=m_{c}\right)$. Hence it soaks up all the leakage of mass acquired by rotation from $\mu=m_{t}$ to $\mu=m_{c}$, leaving $\mathbf{v}_{u}$, by definition orthogonal to $\mathbf{v}_{c}$, with a zero eigenvalue at $\mu=m_{c}$. Any mass for the $u$ state will thus have to rely on leakage from $m_{c}$ by rotation from $\mu=m_{c}$ to $\mu=m_{u}$, just as the mass of the $c$ state did on leakage from $m_{t}$. For the same reason then that $m_{c} / m_{t}$ is small, so $m_{u} / m_{c}$ will be small also.

The same considerations will apply to all fermion types, namely, also to $D$ quarks, charged leptons and neutrinos giving each of the types a hierarchical mass spectrum, except that for neutrinos there may be complications due to an expected see-saw mechanism. In other words, the mass hierarchy is now fully extended to all 3 generations, explaining the observed pattern listed as (A1) above. Further, as noted already before at the end of $\S 1.2$, the R2M2 hypothesis when combined with the assumptions (U) and $(\mathbf{F})$, namely of respectively the universal trajectory and the existence of a high scale fixed point, will explain also the pattern listed above as (A2). What is left unexplained are the points made in (A3), where apart from the great mystery of $m_{d}>m_{u}$, no distinctive pattern emerges among the flavour states.

Next, we turn to the mixing matrices and seek again a geometric explanation for the patterns (B1) and (B2) observed in them above in terms of the curvatures and torsion of the rotation trajectory. Explicitly, the two curvatures are defined as follows. Let us first set up at each point on the trajectory a Darboux triad [17] comprising in the case of a curve on the sphere (i) the radial vector $\boldsymbol{\alpha}$ (ii) the tangent vector $\boldsymbol{\tau}$, (iii) the vector $\boldsymbol{\nu}$ orthogonal to both $\boldsymbol{\alpha}$ and $\boldsymbol{\tau}$, all of unit length. We take the tangent vector $\boldsymbol{\tau}(\mu)$ and differentiate it with respect to the arc length $s$ along the trajectory. This gives us the curvature vector, which must be orthogonal to $\boldsymbol{\alpha}(\mu)$ given that $\boldsymbol{\alpha}$ is of unit length. The component of the curvature vector in the direction of $\boldsymbol{\alpha}$ is then the normal curvature $\kappa_{n}$ while that in the direction $\boldsymbol{\nu}$ is the geodesic curvature $\kappa_{g}$. For a curve on the sphere, the normal curvature is constant, being the curvature of the sphere itself, but the geodesic curvature is a property of the curve and can depend also on the point on it.

To first order in $\delta s$, the Serret-Frenet-Darboux formulae [17] relate two 
neighbouring points $\boldsymbol{\alpha}$ and $\boldsymbol{\alpha}^{\prime}$ on the curve:

$$
\begin{aligned}
\boldsymbol{\alpha}^{\prime}=\boldsymbol{\alpha}(s+\delta s) & =\boldsymbol{\alpha}(s)-\kappa_{n} \boldsymbol{\tau}(s) \delta s+\tau_{g} \boldsymbol{\nu}(s) \delta s \\
\boldsymbol{\tau}^{\prime}=\boldsymbol{\tau}(s+\delta s) & =\boldsymbol{\tau}(s)+\kappa_{n} \boldsymbol{\alpha}(s) \delta s+\kappa_{g} \boldsymbol{\nu}(s) \delta s \\
\boldsymbol{\nu}^{\prime}=\boldsymbol{\nu}(s+\delta s) & =\boldsymbol{\nu}(s)-\kappa_{g} \boldsymbol{\tau}(s) \delta s-\tau_{g} \boldsymbol{\alpha}(s) \delta s
\end{aligned}
$$

where we have kept the geodesic torsion term, which vanishes, to keep track of which terms should be small.

From the formulae in (29), one readily sees that in the limit when the separation between $t$ and $c$ goes to zero, then $\mathbf{v}_{c}$ becomes the tangent $\boldsymbol{\tau}$ to the trajectory at $\mu=m_{t}$ while $\mathbf{v}_{u}$ becomes the normal $\boldsymbol{\nu}$, so that together with $\mathbf{v}_{t}=\boldsymbol{\alpha}\left(\mu=m_{t}\right)$, they constitute the Darboux triad at $\mu=m_{t}$. Similarly, when the separation between $b$ and $s$ goes to zero, the 3 vectors $\mathbf{v}_{b}, \mathbf{v}_{s}, \mathbf{v}_{d}$ become the Darboux triad to the trajectory at $\mu=m_{b}$. So if the separations between $t$ and $c$ and between $b$ and $s$ were both much smaller than the separation between $t$ and $b$, then the CKM matrix would just be the matrix relating the Darboux triads at the two neighbouring points on the trajectory at $\mu=m_{t}$ and $\mu=m_{b}$. To first order in the separation $\delta s$ in arc length between the two points, the Serret-Frenet-Darboux formulae (30) give:

$$
V_{C K M} \sim\left(\begin{array}{lll}
\boldsymbol{\nu} \cdot \boldsymbol{\nu}^{\prime} & \boldsymbol{\nu} \cdot \boldsymbol{\tau}^{\prime} & \boldsymbol{\nu} \cdot \boldsymbol{\alpha}^{\prime} \\
\boldsymbol{\tau} \cdot \boldsymbol{\nu}^{\prime} & \boldsymbol{\tau} \cdot \boldsymbol{\tau}^{\prime} & \boldsymbol{\tau} \cdot \boldsymbol{\alpha}^{\prime} \\
\boldsymbol{\alpha} \cdot \boldsymbol{\nu}^{\prime} & \boldsymbol{\alpha} \cdot \boldsymbol{\tau}^{\prime} & \boldsymbol{\alpha} \cdot \boldsymbol{\alpha}^{\prime}
\end{array}\right) \sim\left(\begin{array}{ccc}
1 & \kappa_{g} \delta s & \tau_{g} \delta s \\
-\kappa_{g} \delta s & 1 & -\kappa_{n} \delta s \\
-\tau_{g} \delta s & \kappa_{n} \delta s & 1
\end{array}\right)
$$

Since our curve is on the unit sphere, the geodesic torsion $\tau_{g}$ vanishes and the normal curvature $\kappa_{n}$ is unity. It then immediately follows that the two corner elements $V_{u b}, V_{t d}$, being of order at least $\delta s^{2}$, have to be much smaller than the other nondiagonal elements $V_{c b}, V_{t s}$ and $V_{u s}, V_{c d}$ of order $\delta s$, giving thus the pattern (B1) in the CKM matrix already noted.

In reality, of course, the separation between the two heaviest generations is not small for either $U$ or $D$ compared with the separation between $t$ and $b$, so that the approximations made in the paragraph above to derive (31) do not actually apply. Nevertheless, it gives us a qualitatively correct picture for what happens to the CKM matrix elements in relation to the behaviour of the rotation trajectory. In particular, it is now easy to see why the CKM elements in the last row and column are hierarchical in a manner so reminiscent of the mass hierarchy, a similarity often remarked on. In the present language, say for $U$ quarks, if we interpret the separation $\delta s$ as the displacement from $t$ to 
$c$, and from $t$ to $u$ respectively, the relations (23) would give us:

$$
\begin{aligned}
& m_{c} \sim m_{t}\left|\boldsymbol{\alpha}^{\prime} \cdot \boldsymbol{\tau}\right|^{2} \sim m_{t}(\delta s)^{2} \\
& m_{u} \sim m_{t}\left|\boldsymbol{\alpha}^{\prime} \cdot \boldsymbol{\nu}\right|^{2} \sim 0
\end{aligned}
$$

the last term being 0 as the geodesic torsion vanishes. In other words, both the elements $V_{c b}, V_{t s}$ and the mass ratios $m_{c} / m_{t}, m_{s} / m_{b}$ come from the bending of the trajectory along the sphere (normal curvature) and hence are much larger than respectively the corner elements $V_{u b}, V_{t d}$ and the mass ratios $m_{u} / m_{t}, m_{d} / m_{b}$ both of which can come only from a twist in the trajectory, necessarily of second or higher order in the separation since the trajectory has no geodesic torsion.

The same considerations applied to the leptons will show that in the PMNS matrix the corner element $U_{e 3}$ governed by the twist of the trajectory will be much smaller than either of the elements $U_{e 2}$ and $U_{\mu 3}$ governed by the bend of the trajectory, which is indeed as observed. In other words, the pattern (B1) is now fully explained.

Turning next to the pattern (B2), we observe that since the normal curvature here is constant as already noted, the corresponding elements 23,32 in the mixing matrices will just be proportional in magnitude to the separation between the two states labelled by 2 and 3 . Hence, given the much smaller mass ratio $m_{\nu_{3}} / m_{\mu}$ compared with $m_{s} / m_{t}, m_{c} / m_{b}$, it follows that $U_{\mu 3}$ in the PMNS matrix would be much larger than the corresponding elements $V_{t s}, V_{c b}$ in the CKM matrix. The fact that the quarks, being located nearer the high scale fixed point postulated in the assumption (F) above, will be moving more slowly along the trajectory than the leptons will only serve to enhance the above difference. This then accounts for half of the pattern (B2) listed.

As for the elements of the mixing matrices proportional to the geodesic curvature $\kappa_{g}$, namely $V_{u s}, V_{c d}$ in $V_{C K M}$ and $U_{e 2}$ in $U_{P M N S}$, they depend both on the trajectory and on the point on it, and cannot therefore be deduced simply from the rotation hypothesis. One notes, however, that they can be very different both in magnitude and in scale-dependence from the mixing elements proportional to the normal curvature considered in the preceding paragraph, as they indeed seem to be, as observed in (B2). As to in what way they differ is model-dependent and will have to be left to model-builders. An explicit example is given in [15, 14].

One important property of the $3-\mathrm{G}$ mixing matrix not yet mentioned with no analogue in the 2-G case is of course the Kobayashi-Maskawa CP-violating phase [7]. In the treatment above of R2M2, the mass matrix (22) factorizes 
into a vector $\boldsymbol{\alpha}$ which has been taken tacitly as real, although this need not be the case. If the elements of $\boldsymbol{\alpha}$ are taken complex but their relative phases do not change with scale, then since only lengths and inner products of vectors are of physical interest, the results would be no different from what were obtained above with real $\boldsymbol{\alpha}$. The mixing matrices, in particular, will still have no KM CP-violating phase. Only when the elements of $\boldsymbol{\alpha}$ are allowed to have relative phases changing with scale can one obtain a nonzero $\mathrm{KM}$ phase in the mixing matrix in the rotation scheme. Such a scenario is not easy to implement, given no empirical indication of such phase changes in $\boldsymbol{\alpha}$. Besides, in all the models so far constructed, the vector $\boldsymbol{\alpha}$ turns out, for reasons that are well understood, always to have a constant phase, i.e. effectively real. At this stage of the presentation, therefore, the absence of a KM phase in the mixing matrix should be counted as a major failure of the R2M2 hypothesis. However, in the next section, it will be shown how the R2M2 hypothesis contains in itself a quite intriguing mechanism for removing this shortcoming while offering at the same time a solution to the long-standing strong CP problem in QCD.

\subsection{Does it work for $3-\mathrm{G}$ too?}

It is seen in the preceding subsection that the R2M2 hypothesis when supplemented by the assumptions $(\mathbf{U})$ and $(\mathbf{F})$ does seem to go quite a long way towards explaining the qualitative features of both the fermion mass spectrum and the quark and lepton mixing matrices. One needs however further to ask, as one did for the 2-G case, whether it really works in practice when applied to the physical system at hand. One can address this question in two ways. One can devise a model trajectory with the appropriate features depending perhaps on some parameters, then try to fit experimental data with it and see whether one obtains a reasonable description. Or else, one can proceed in a more model-independent manner, as one did in the 2-G case before in $§ 1.2$, starting with the data and see whether they can be fitted with some sensible trajectory.

The first approach was first tried, indeed historically even before the R2M2 hypothesis was properly formulated. A phenomenological model (DSM) [13] was constructed based on some theoretical ideas which led to a renormalizaton group equation for $\boldsymbol{\alpha}$, with the property that it possesses a rotational fixed point at $\mu=\infty$, satisfying thus assumption (F) as desired. That the trajectory is universal, assumption (U), was inserted as input from experiment. The resulting trajectory depends on 3 adjustable real parameter, 
explicitly a coupling parameter governing the speed of rotation with respect to scale, plus two integration constants. These 3 parameters were varied to fit the then existing data [19]. One obtained [12] reasonable values for the mass ratios, and good agreement for all entries of the CKM matrix, as well as those of the PMNS matrix, except for the solar neutrino angle $U_{e 2}$ depending on the geodesic curvature sensitive to the details of the rotation trajectory, and for the light quark masses the exact interpretation of which is complicated by confinement. This was not bad for 3 parameters, and thought at first to be a success of the DSM model.

It was soon realized, however, that the agreement with experiment obtained was not due so much to the validity of the model which, being intended to be merely phenomenological, contained some rather arbitrary features, but rather to the general concept of rotation, namely what one now calls the R2M2 hypothesis. What is relevant for the present is thus not so much the premises on which this model (DSM) was built but the fact that with a model trajectory possessing the envisaged features, one was able to obtain already quite a sensible description of the then available data, which can thus be taken tentatively as evidence that R2M2 works even in the 3-G case.

The more direct approach for testing the R2M2 hypothesis, starting with the data to deduce the shape of the trajectory, as was done in Figure 3 for the 2-G case, was also attempted previously in [5, 20]. But, at that time, the data did not yield much more than what was contained already in Figure 3. and one did not know how to take account of CP-violation. With now considerably more accurate data, and some improvements in technique now available, the analysis will be repeated in $\S 2.2$ below after the problem of the KM CP-violating phase is resolved so that the effects of this phase can be properly incorporated as they should be. We anticipate the result [21] in stating that it is positive, meaning that the R2M2 hypothesis is found to be still consistent with the existing data of much improved accuracy.

\section{New Shoots}

The situation as reviewed in the last section, though reasonably satisfying in reproducing sensibly the effects one started out to explain, still needs to be viewed with caution in that the effects "explained" were known before one started, and so in constructing the answer, namely the R2M2 hypothesis, as it is now called, one might have simply doctored the answer to fit the desired outcome. To be more convinced of its validity, one can proceed in two 
ways. One can go back to theory and seek a justification there for the R2M2 hypothesis, by devising, for example, a viable model behind the standard model as outlined above, which will produce the R2M2 automatically as a consequence. Or else, one can go forward and seek new premises on which to apply the R2M2 hypothesis so as to resolve problems or make predictions for which it was originally not devised. An effort along the first direction is reported in [11, 14] as already mentioned. Some efforts along the second direction are what will be reviewed below.

\section{$2.1 \quad$ A tale of two CP's}

For an example of a problem to attack using R2M2 outside its original remit of mass hierarchy and mixing, we have long had our eyes on the strong CP problem in QCD. To see why, let us first recall what is meant by the strong $\mathrm{CP}$ problem.

The "strong CP problem" is a long-standing problem in QCD, which has been with us for over forty years [22]. There, gauge invariance in colour allows in principle a term in the action of the form:

$$
\mathcal{L}_{\theta}=-\frac{\theta}{64 \pi^{2}} \epsilon^{\mu \nu \rho \sigma} F_{\mu \nu} F_{\rho \sigma}
$$

for arbitrary $\theta$. This term, which is of topological origin, violates $\mathrm{CP}$ and can lead to a large electric dipole moment for the neutron. Experimentally, however, the existing limit of the neutron dipole moment is now $2.7 \times 10^{-26} \mathrm{e}$ $\mathrm{cm}$ [23] which means that the angle $\theta$ in (33) has, for some unknown reason, to be of the order $3 \times 10^{-10}$ or less [22].

The favourite theoretical suggestion for resolving this strong CP problem is the axion theory [24, 25], but the axion has been looked for experimentally since soon after the problem was recognized but so far has not been found. Hence, any new insight into the problem should be more than welcome.

The theta-angle term (33) above is of the same form as the change in the Jacobian in the Feynman integral when one performs a chiral transformation on a fermion field appearing as an integration variable. Hence, in a theory with $N$ flavours of quarks, a chiral transformation, thus:

$$
\psi \longrightarrow \exp \left(i \alpha \gamma_{5}\right) \psi
$$

on each quark flavour will yield a theta-angle term modified to:

$$
\theta \longrightarrow \theta+2 \sum_{F} \alpha_{F}
$$


Clearly, this can be made to vanish by a judicious choice of $\alpha_{F}$. Since a mere change of integration variables cannot change the value of the integral which contains the physics, it would seem that the effect of the theta-angle term can thereby be eliminated. However, the above chiral change of variables would also affect the integrand, in particular the mass term of the quarks if they are massive, and it will make their mass parameters in general complex:

$$
\begin{aligned}
m \bar{\psi} \psi & =m \bar{\psi} \frac{1}{2}\left(1+\gamma_{5}\right) \psi+m \bar{\psi} \frac{1}{2}\left(1-\gamma_{5}\right) \psi \\
& \rightarrow m \exp (2 i \alpha) \bar{\psi} \frac{1}{2}\left(1+\gamma_{5}\right) \psi+m \exp (-2 i \alpha) \bar{\psi} \frac{1}{2}\left(1-\gamma_{5}\right) \psi
\end{aligned}
$$

leading again to $\mathrm{CP}$-violation. Only when there is a quark with zero mass can the theta-angle term be eliminated without having to pay the penalty, a fact already recognized again for a long time as a possible solution to the strong $\mathrm{CP}$ problem. Unfortunately, none of the quarks known can be assigned a zero mass in experiment, and so the problem remains.

The reason one thought right away [26, 27, 28] that R2M2 might be relevant for the strong $\mathrm{CP}$ problem is the fact noted above in $\S 1.4$ that R2M2 keeps chiral invariance while allowing all quark masses to be nonzero. This would seem to mean that chiral transformations can be performed to eliminate any theta-angle term in the action and yet give non-zero masses for all quarks as experiment seems to demand. That observation is however incomplete in that even in the usual scenario, the problem is not with the elimination of the theta-angle term itself by a chiral transformation, which can always be performed, but with the effect of the transformation on the rest of the action, which can acquire thereby $\mathrm{CP}$-violations elsewhere. It would therefore be necessary in the R2M2 scenario also to examine how the rest of the action is affected by the chiral transformation, and whether or not the CP-violation is transmitted somewhere else. To do so, one will need to perform the chiral transformation explicitly on the 3-component quark field $\boldsymbol{\psi}$ (for 3 generations) and then to follow through its effect down to the level of measured quantities in the R2M2 scenario.

This has now been done as follows [29]. One starts by considering first the R2M2 mass matrix (2) at some chosen value of $\mu$. It has two independent vectors with zero eigenvalues orthogonal to the rotating vector $\boldsymbol{\alpha}(\mu)$, which may be taken as first, the normalized tangent vector $\boldsymbol{\tau}(\mu)$ to the trajectory which is orthogonal to $\boldsymbol{\alpha}(\mu)$ since the latter remains of unit length, and second, the normalized normal vector $\boldsymbol{\nu}(\mu)$ orthogonal to both $\boldsymbol{\alpha}(\mu)$ and $\boldsymbol{\tau}(\mu)$. These 3 vectors $\boldsymbol{\alpha}(\mu), \boldsymbol{\tau}(\mu)$, and $\boldsymbol{\nu}(\mu)$, form the Darboux triad to the trajectory at $\mu$, as was noted already in $\S 1.5$. Now, both the vectors 
$\boldsymbol{\tau}(\mu)$ and $\boldsymbol{\nu}(\mu)$ being eigenvectors of $m(\mu)$ with zero eigenvalues, a chiral transformation on the quark field corresponding to either component can be performed to eliminate the theta-angle term at $\mu$, yet leaving $m(\mu)$ invariant and hence hermitian. Consider however the mass matrix $m(\mu+d \mu)$ at a neighbouring point $\mu+d \mu$ on the trajectory, which is given explicitly in terms of $\dot{\boldsymbol{\alpha}}(\mu)$ as:

$$
m(\mu+d \mu)=m_{T}(\boldsymbol{\alpha}(\mu)+\dot{\boldsymbol{\alpha}}(\mu) d \mu)(\boldsymbol{\alpha}(\mu)+\dot{\boldsymbol{\alpha}}(\mu) d \mu)^{\dagger} .
$$

One sees then that $\boldsymbol{\tau}(\mu)$, which is proportional to $\dot{\boldsymbol{\alpha}}(\mu)$, is not an eigenvector of $m(\mu+d \mu)$ with zero eigenvalue, so that $m(\mu+d \mu)$ will not be left hermitian by a chiral transformation performed on $\boldsymbol{\psi}$ along the component corresponding to the tangent $\boldsymbol{\tau}(\mu)$. Only a chiral transformation along the normal component $\boldsymbol{\nu}(\mu)$ at $\mu$ to eliminate the theta-angle term will leave both $m(\mu)$ and $m(\mu+d \mu)$ hermitian.

Iterating then the procedure, eliminating the theta-angle term at every $\mu$ by a chiral transformation on $\boldsymbol{\nu} \cdot \boldsymbol{\psi}$, the component along the normal $\boldsymbol{\nu}(\mu)$ of the quark field, will leave the mass matrix $m(\mu)$ hermitian all along the trajectory.

To be explicit, let us choose a reference frame in 3-D generation space such that at $\mu=\infty$, we have:

$$
\boldsymbol{\alpha}(\infty)=\boldsymbol{\alpha}_{0}=\left(\begin{array}{l}
1 \\
0 \\
0
\end{array}\right) ; \boldsymbol{\tau}(\infty)=\boldsymbol{\tau}_{0}=\left(\begin{array}{l}
0 \\
1 \\
0
\end{array}\right) ; \boldsymbol{\nu}(\infty)=\boldsymbol{\nu}_{0}=\left(\begin{array}{l}
0 \\
0 \\
1
\end{array}\right)
$$

and define a rotation $A(\mu)$ such that:

$$
\boldsymbol{\alpha}(\mu)=A(\mu) \boldsymbol{\alpha}_{0} ; \boldsymbol{\tau}(\mu)=A(\mu) \boldsymbol{\tau}_{0} ; \quad \boldsymbol{\nu}(\mu)=A(\mu) \boldsymbol{\nu}_{0}
$$

The chiral transformation needed to eliminate the theta-angle term from the action at scale $\mu$ can then be represented as:

$$
P(\mu)=A(\mu) P_{0} A^{-1}(\mu), \quad P_{0}=\left(\begin{array}{ccc}
1 & 0 & 0 \\
0 & 1 & 0 \\
0 & 0 & e^{-i \theta \gamma_{5} / 2}
\end{array}\right)
$$

Effecting such a chiral transformation on the quark field at every $\mu$ would then remove the theta-angle term entirely and leave the QCD action CPinvariant and the mass matrix hermitian at every $\mu$. 
Notice, however, that since $\boldsymbol{\alpha}$ rotates, the normal $\boldsymbol{\nu}(\mu)$ will also change its direction as a function of $\mu$. Hence, to iterate the procedure at $\mu+d \mu$ so as to eliminate the theta-angle term there while ensuring the hermiticity of the mass matrix at the next neighbouring point too, one has first to undo the previous chiral transformation performed at $\mu$ before, and then effect again the chiral transformation at $\mu+d \mu$, namely to apply the operator:

$$
P(\mu+d \mu) P^{-1}(\mu)
$$

to the quark fields, in order to obtain the desired result. This operator (41) acts thus as a kind of parallel transport, detailing effectively what is meant by the same or parallel (chiral) phases at two neighbouring points along the trajectory, and hence, by iteration, at any two points a finite distance apart.

To see how these chiral transformations will affect the conclusions above of R2M2 on quark masses and mixing, let us start first with just one type of quarks, say, the $U$-type quarks, i.e. $t, c, u$. We shall label the Darboux triad $(\boldsymbol{\alpha}, \boldsymbol{\tau}, \boldsymbol{\nu})$ at $\mu=m_{t}$ as $\left(\boldsymbol{\alpha}_{U}, \boldsymbol{\tau}_{U}, \boldsymbol{\nu}_{U}\right)$. The state vector of $t$, i.e. $\mathbf{v}_{t}$, or just $\mathbf{t}$ for short, is defined as $\boldsymbol{\alpha}_{U}$ and the state vectors $\mathbf{c}, \mathbf{u}$ are to be orthogonal to it and are themselves mutually orthogonal. It follows therefore that the dyad $\mathbf{c}, \mathbf{u}$ is related to the dyad $\boldsymbol{\tau}_{U}, \boldsymbol{\nu}_{U}$ by a rotation in the plane orthogonal to $\mathbf{t}$, thus:

$$
\mathbf{c}=\Omega_{U} \boldsymbol{\tau}_{U} ; \mathbf{u}=\Omega_{U} \boldsymbol{\nu}_{U}
$$

with

$$
\Omega_{U}=\left(\begin{array}{ccc}
1 & 0 & 0 \\
0 & \cos \omega_{U} & -\sin \omega_{U} \\
0 & \sin \omega_{U} & \cos \omega_{U}
\end{array}\right),
$$

$\omega_{U}$ being the angle between $\mathbf{c}$ and $\boldsymbol{\tau}_{U}$. This angle is small but nonzero since, according to our prescription above, $\mathbf{c}=\mathbf{v}_{c}$ is the vector which is orthogonal to $\boldsymbol{\alpha}\left(\mu=m_{t}\right)$ and lies on the plane containing both the vectors $\boldsymbol{\alpha}\left(\mu=m_{t}\right)$ and $\boldsymbol{\alpha}\left(\mu=m_{c}\right)$, while $\boldsymbol{\tau}_{U}$ is the tangent to the trajectory at $\mu=m_{t}$; it is thus a measure of how much $\boldsymbol{\alpha}(\mu)$ has rotated from $\mu=m_{t}$ to $\mu=m_{c}$. Suppose we wish again to evaluate the mass of the $c$ quark in the rotation scenario as we did before but incorporating now the above procedure for eliminating the theta-angle term, we shall have to take the $c$ quark field $\psi_{c}\left(\mu=m_{t}\right)=\mathbf{c} \cdot P\left(m_{t}\right) \boldsymbol{\psi}$, but it will now have to be parallelly transported by (41) to $\mu=m_{c}$, giving $\psi_{c}\left(\mu=m_{c}\right)=\mathbf{c} \cdot P\left(m_{c}\right) \boldsymbol{\psi}$. The mass term also, according to (36) above, will now appear at $\mu=m_{c}$ as:

$$
m_{T} \overline{\boldsymbol{\psi}} P\left(m_{c}\right) \boldsymbol{\alpha}\left(m_{c}\right) \boldsymbol{\alpha}^{\dagger}\left(m_{c}\right) P\left(m_{c}\right) \boldsymbol{\psi}
$$


where the operators $P\left(m_{c}\right)$ can in fact be omitted since the vector $\boldsymbol{\alpha}\left(m_{c}\right)$ is invariant under $P\left(m_{c}\right)$. What interests us here as far as the $c$ mass is concerned, according to the analysis in, for example, [28], is the diagonal contribution from the $c$ quark, namely:

$$
\begin{aligned}
& m_{T} \overline{\boldsymbol{\psi}} P\left(m_{c}\right) \mathbf{c c}^{\dagger} \boldsymbol{\alpha}\left(m_{c}\right) \boldsymbol{\alpha}^{\dagger}\left(m_{c}\right) \mathbf{c c}^{\dagger} P\left(m_{c}\right) \boldsymbol{\psi} \\
& \quad=m_{T}\left|\mathbf{c} \cdot \boldsymbol{\alpha}\left(m_{c}\right)\right|^{2} \bar{\psi}_{c}\left(\mu=m_{c}\right) \psi_{c}\left(\mu=m_{c}\right)
\end{aligned}
$$

giving then the $c$ mass as:

$$
m_{c}=m_{T}\left|\mathbf{c} \cdot \boldsymbol{\alpha}\left(m_{c}\right)\right|^{2},
$$

i.e., exactly the same as before (23) when no consideration was given to the elimination of the theta-angle term. The same conclusions will apply also to $m_{u}$. One sees therefore that so long as there is only one type of quarks, one can always manage, with a rotating factorizable mass matrix, to eliminate any theta-angle term so as to maintain CP-conservation, while keeping the mass matrix hermitian, and having at the same time hierarchical but nonzero masses for all the quarks.

What happens, however, when there are both up-type and down-type quarks? In that case, the two types can be coupled by the weak current via the CKM mixing matrix, and one has again to follow through the preceding arguments and trace out the consequence of eliminating the theta-angle term. To do so, let us denote the state vectors of the $U$-type quarks defined above at $\mu=m_{t}$ together as:

$$
V_{U}=(\mathbf{t}, \mathbf{c}, \mathbf{u})=\left(\begin{array}{ccc}
t_{1} & c_{1} & u_{1} \\
t_{2} & c_{2} & u_{2} \\
t_{3} & c_{3} & u_{3}
\end{array}\right)
$$

and similarly the state vectors of the $D$-type quarks defined at $\mu=m_{b}$ as:

$$
V_{D}=(\mathbf{b}, \mathbf{s}, \mathbf{d})=\left(\begin{array}{lll}
b_{1} & s_{1} & d_{1} \\
b_{2} & s_{2} & d_{2} \\
b_{3} & s_{3} & d_{3}
\end{array}\right)
$$

where in the notation introduced above, one has from rotation:

$$
\begin{aligned}
& V_{U}=A_{U} \Omega_{U} ; \quad A_{U}=A\left(\mu=m_{t}\right) \\
& V_{D}=A_{D} \Omega_{D} ; \quad A_{D}=A\left(\mu=m_{b}\right) .
\end{aligned}
$$


What is of interest is the relative orientation of $V_{U}$ and $V_{D}$, the matrix of inner products between the state vectors of the $U$-type and $D$-type quarks being the starting point for the CKM mixing matrix we seek. In order to compare the orientation of the state vectors of one type to those of the other, the two types being defined as they are at two different scales, one needs first to parallelly transport the (chiral) phase of each to the same scale, say $X$, before one can make due comparison, thus:

$$
\begin{aligned}
& P_{X} P_{U}^{-1} V_{U}=P_{X}\left(A_{U} P_{0}^{-1} A_{U}^{-1}\right)\left(A_{U} \Omega_{U}\right)=\tilde{V}_{U} \\
& P_{X} P_{D}^{-1} V_{D}=P_{X}\left(A_{D} P_{0}^{-1} A_{D}^{-1}\right)\left(A_{D} \Omega_{D}\right)=\tilde{V}_{D} .
\end{aligned}
$$

Hence we obtain the CKM matrix in this scenario as:

$$
V_{C K M}=\tilde{V}_{U}^{-1} \tilde{V}_{D}=\left(\Omega_{U}^{-1} P_{0} \Omega_{U}\right) V_{U}^{-1} V_{D}\left(\Omega_{D}^{-1} P_{0}^{-1} \Omega_{D}\right),
$$

independent of the chosen scale $X$, as expected. Since only the left-handed quark fields are involved in the weak current, the chiral phase exp $-i \theta \gamma_{5} / 2$ in $P_{0}$ has been replaced by the phase factor exp $-i \theta / 2$.

Notice that the factor $V_{U}^{-1} V_{D}$ would be the CKM matrix if there were no theta-angle term to contend with, and it would be a real matrix with no $\mathrm{CP}$-violating phase if we started with a real $\boldsymbol{\alpha}$ as we have done. It will be convenient to refer to it as the $U D$ matrix. By insisting on the chiral transformations to eliminate the theta-angle term throughout, one has injected some new phases into the mixing matrix elements, and hence the possibility of CP-violation, which will indeed be the case if the phases introduced by the said chiral transformations cannot be removed by any changes in phase of the physical quark states. Indeed, if we were to put both $\omega_{U}=0$ and the corresponding $D$-type angle $\omega_{D}=0$ in (51), one would have obtained vanishing values for the Jarlskog invariant [30] and no CP-violation. The reason is clear, since in that case the vector $\mathbf{u}$ would coincide with the normal vector $\boldsymbol{\nu}$ at $\mu=m_{t}$, the component of the quark field on which the chiral transformation is performed, and the effect on the CKM matrix would be the same as that of changing the phase of the physical $u$ field, which is arbitrary; and similarly for the phase of the physical $d$ field. If one were to calculate the Jarlskog invariant from (51) in this case, the phases would cancel and one obtains a zero value. Since, however, $\omega_{U}$ and $\omega_{D}$ are nonzero by virtue of the rotation as explained above, this cancellation has now no reason to occur and one has in general a nonvanishing Jarlskog invariant and $\mathrm{CP}$-violations as the result. 
One concludes therefore that even if starting with a real vector $\boldsymbol{\alpha}$ in the factorized mass matrix (2) in R2M2, the presence of a theta-angle term in the action and the process of eliminating it through a chiral transformation as detailed above will automatically give rise to CP-violations in the CKM matrix. But will this yield Jarlskog invariants and CP-violating effects of the order observed in experiment? We recall that the strong CP angle $\theta$ from which this effect is supposed to originate can take in principle any arbitrary value and so should be taken without prejudice as of order unity, whereas the measured value of the Jarlskog invariant is of order $3 \times 10^{-5}$, so that a suppression by about 4 orders of magnitude is required in the process of transmitting the CP-violating effects from the strong sector to the weak sector via rotation. This is possible, so long as the rotation is relatively slow as is envisaged. To see whether it is indeed the case, one can evaluate the Jarlskog invariant for (51) with, for example, its $2 \times 2$ submatrix labelled by the 2 heaviest states $t, c$ and $b, s$. One obtains then an explicit expression for $J$ in terms of $\theta, \omega_{U}, \omega_{D}$ and elements of the $U D$ matrix $V_{U}^{-1} V_{D}$. The angles $\omega_{U}$ and $\omega_{D}$, one has already noted to be of order $\epsilon$, the angle rotated by the vector $\boldsymbol{\alpha}$ from the scale of the heaviest to that of the second generation. Further, from an earlier analysis of the rotation picture [31], as reviewed in $\S 1.5$ above, one has learned that the CKM matrix elements $V_{t s}, V_{c b}, V_{c d}, V_{u s}$ proportional to the curvatures of the rotation trajectory are all of order $\epsilon$, while the corner elements $V_{t d}, V_{u b}$ proportional to its geodesic torsion are of order at least $\epsilon^{2}$. This is not to say, of course, that all four elements of order $\epsilon$ need be of the the same size for, as already remarked before, the two curvatures, normal and geodesic, can have quite different values. But, for the order-of-magnitude estimate generic to the rotation scheme aimed for at the moment, one can ignore such details specific to a particular trajectory, and just substitute the above estimates into the formula for $J$. One finds then that $J$ is of order $\epsilon^{4}$ and proportional to $\sin (\theta / 2)$. An estimate for the value of $\epsilon$ can be obtained from the rotation formula (46) for the mass ratio of the second generation to the heaviest, leading to $\epsilon \sim 0.08$ for $m_{c} / m_{t}$, and $\epsilon \sim 0.15$ for $m_{s} / m_{b}$. This then gives an order-of-magnitude estimate for the Jarlskog invariant as:

$$
J \sim \sin (\theta / 2) \times 10^{-4}
$$

which is quite consistent with the experimentally measured value [18] of $\sim 3 \times 10^{-5}$ for a strong CP angle $\theta$ of order unity 5

\footnotetext{
${ }^{5}$ In the above analysis, the vector $\boldsymbol{\alpha}$ is all along tacitly taken to be real, whereas it can, in principle, be complex and, as already noted in $\S 1.5$, if the relative phases of its
} 
One knows of course, whether in the rotation framework or otherwise, that once given the small values observed in experiment for mixing angles involving the two heaviest states $t$ and $b$, it will follow already that the CPviolating effects of the KM phase will be small, since it is known that for two generations there is no CP-violation, which can thus arise only through mixing with $t$ and $b$. A priori, however, one can give no actual estimate for the size of the effects, not knowing how or where the KM phase originates. The difference with the scheme here is that, first, having traced the origin of the KM phase via rotation back to the strong sector, one can give now an actual estimate for $J$, and second, since the rotation relates also the mixing angles of fermions to their hierarchical masses, as explained in $\S 1.3$ above, the estimate can be derived with only mass ratios as inputs, and no knowledge of the mixing angles being required at all. In other words, the rotation has indeed managed by itself, in transmitting the CP-violating effects from the strong to the weak sector, the suppression by some four orders of magnitude noted above as needed to bring them correctly down to the order observed in experiment.

If one is willing to supply more empirical information such as the experimentally measured values of the mixing angles as inputs to the rotation scheme, then one would obtain more accurate estimates of the Jarlskog invariant from an assumed value of order unity for the theta-angle, or conversely an estimate of the theta-angle from the measured value of the Jarlskog invariant. This will be done in the following subsection. Before we do so, however, let us first pause for a moment to take stock of the new situation.

As far as the R2M2 hypothesis is concerned, the result represents, first, the removal of a major shortcoming in the R2M2 framework as reviewed in the last section and noted at the end of $\S 1.5$, which is significant for the further development of the framework. And secondly, but equally significantly, it provides a first example of the R2M2 idea applied, successfully it seems, to problems outside the area for which it was originally devised. We recall that the R2M2 hypothesis was originally conceived as a means for explaining fermion mass hierarchy and mixing. Here it has been applied to yield a solution for the long-standing strong-CP problem in QCD, which has at first sight little to do with the mass spectrum and mixing patterns of fermion states. And, as far as one can see, no new assumption has been made in deriving the new result. It is, therefore, in a sense a prediction, and adds

components vary with scale, then they can lead to CP-violating phases in the CKM matrix as well. However, now that it is seen that even for a real $\boldsymbol{\alpha} \mathrm{CP}$-violations can result from a theta-angle term, the other possibility has lost much of its appeal. 
much to the credibility of the R2M2 hypothesis. Thirdly, this result, relying as it does on the unusual property of R2M2 in giving nonzero quark masses despite maintaining strict chiral invariance in the action, opens the door to other problems of a similar sort. In particular, one has in mind the problem of chiral symmetry breaking in QCD, for which R2M2 would now suggest a very novel picture, namely, that chiral symmetry is never really broken, the deviations from symmetry one sees being only due to the physical fermion states being wrongly identified as eigenstates of the nominal mass matrix derived from the chiral invariant action rather than correctly as eigenstates of the physical mass matrix obtained by a truncation thereof, as detailed in $§ 1.4$. The question of whether this picture for chiral symmetry breaking can hold or not is being worked on. If answered in the affirmative, it would obviously add further to the credibility of R2M2.

Furthermore, this new result on $\mathrm{CP}$, in linking the theta-angle term in the QCD action to the CP-violating phase in the CKM matrix, brings out a point of considerable theoretical interest way beyond the original phenomenological remits of the R2M2 hypothesis. Indeed, if the above result were to be adopted, it would change our conception of the CP problem altogether. It used to be that one has, on the one hand, a strong CP problem with its origin in topology that one does not quite know how to solve and, on the other, what one may call a weak CP problem of the KM phase which one knows ought to be there but not whence its origin nor how to estimate its size. The two sides were separate and apparently totally unrelated. But now, what R2M2 seems to be telling us is that the two CP problems, strong and weak, are actually one and the same phenomenon. The unwanted theta-angle term in the QCD action, which invariance principles tell us ought to be there, can be eliminated by a chiral transformation of integration variables, or in other words by just a redefinition of what the $\mathrm{CP}$ operation means, at no other cost, it seems, than introducing a KM phase in the CKM matrix, which one would want in any case. This means, of course, that the KM phase is now traced to a topological origin, i.e. same as the theta-angle term, and the relation between the two will yield an estimate for its size, as it seems correctly to have done. Turning the argument around, this means also that the existence of the KM phase as observed in experiment is telling us, via rotation and the theta-angle term, about the topology of the QCD world, and can even give us an estimate of the value of the theta-angle, which, as seen, will be of order unity. Even apart from the phenomenology, this seems altogether a quite enchanting theoretical picture to have emerged. 


\subsection{Sequel to "Does it work for 3-G too'?"}

Having now understood how the KM CP-violating phase is to appear in the R2M2 scheme, which was the missing link in $\S 1.6$, we are now in a position to re-examine the question whether the recent data of accuracy much superior to those used in earlier attempts [5] are still consistent with the R2M2 hypothesis. In other words, in the same spirit as Figure 3 in the planar approximation of $\$ 1.2$, we wish to know whether the new full 3-generation data can be fitted with a smooth trajectory for $\boldsymbol{\alpha}(\mu)$.

The R2M2 scheme has $\boldsymbol{\alpha}$ as a fundamental object, and the state vectors are derived from this. To test the R2M2 hypothesis, however, we shall start from real orthonormal state vectors and use experimental data to find a consistent trajectory of $\boldsymbol{\alpha}$. We first choose the $U$-type quarks to have state vectors:

$$
\begin{aligned}
& \mathbf{v}_{u}=\left(\begin{array}{ll}
1, & 0,0
\end{array}\right)^{\dagger}, \\
& \mathbf{v}_{c}=\left(\begin{array}{ll}
0, & 1,0
\end{array}\right)^{\dagger}, \\
& \mathbf{v}_{t}=\left(\begin{array}{lll}
0, & 0,1
\end{array}\right)^{\dagger},
\end{aligned}
$$

which we are free to do, and which are real as explained above. Once we have chosen a $U D$ matrix we can use it to define the $D$-type quark state vectors. There are no explicit physical constraints on the $U D$ matrix so we are free to choose any orthogonal matrix.

From the mass leakage mechanism, $\boldsymbol{\alpha}\left(\mu=m_{t}\right)=\mathbf{v}_{t}$, and similarly $\boldsymbol{\alpha}(\mu=$ $\left.m_{b}\right)=\mathbf{v}_{b}$. The leakage mechanism then fixes $\boldsymbol{\alpha}_{c}$ and $\boldsymbol{\alpha}_{s}$ in terms of the quark masses:

$$
\begin{aligned}
& \boldsymbol{\alpha}_{c}=\sqrt{m_{c} / m_{t}} \mathbf{v}_{c}+\sqrt{1-m_{c} / m_{t}} \mathbf{v}_{t}, \\
& \boldsymbol{\alpha}_{s}=\sqrt{m_{s} / m_{b}} \mathbf{v}_{s}+\sqrt{1-m_{s} / m_{b}} \mathbf{v}_{b} .
\end{aligned}
$$

The two vectors $\mathbf{v}_{t}$ and $\mathbf{v}_{c}$ define a plane. All that the mass ratio $m_{u} / m_{t}$

tells us about $\boldsymbol{\alpha}_{u}$ is the angle which it makes with this plane. We can thus restrict $\boldsymbol{\alpha}_{u}$, and similarly $\boldsymbol{\alpha}_{d}$, to lie somewhere on a line, parametrized by $t \in[0,2 \pi):$

$$
\begin{aligned}
& \boldsymbol{\alpha}_{u}=\sqrt{m_{u} / m_{t}} \mathbf{v}_{u}+\sqrt{1-m_{u} / m_{t}} \mathbf{v}_{c} \cos (t)+\sqrt{1-m_{u} / m_{t}} \mathbf{v}_{t} \sin (t) \\
& \boldsymbol{\alpha}_{d}=\sqrt{m_{d} / m_{b}} \mathbf{v}_{d}+\sqrt{1-m_{d} / m_{b}} \mathbf{v}_{s} \cos (t)+\sqrt{1-m_{d} / m_{b}} \mathbf{v}_{b} \sin (t)
\end{aligned}
$$


where we will choose the signs of the square roots so that $\boldsymbol{\alpha}_{u, d}$ has a positive projection onto $\mathbf{v}_{u, d}$. The restrictions on $\boldsymbol{\alpha}_{i}$ for the charged leptons were found in an analogous way, replacing $(u, c, t)$ with $(e, \mu, \tau)$. The neutrinos place only very weak restrictions on the trajectory of $\boldsymbol{\alpha}$ since their masses, and the PMNS matrix elements, are not well measured.

Before we give any results we will take a moment to discuss their presentation. The state vectors and $\boldsymbol{\alpha}$ are in $\mathbb{R}^{3}$ and of unit length so take values on the surface of a unit sphere. We will represent their positions by stereographically projecting onto $\mathbb{R}^{2}$. It turns out that $\boldsymbol{\alpha}$ does not need to rotate very far from $\mu=m_{t}$ to $\mu=m_{e}$ so most of the action happens in a small area on the sphere. We have chosen the south pole of the projection to be at the position of $\mathbf{v}_{\tau}$. This means that there will not be much distortion introduced by the stereographic projection; geodesics in this region on the sphere will be almost straight lines on the plane. Figure 4 shows the unit sphere with the region we will be interested in enclosed in a box. The curve within the box shows the best fit line we find and the point shows the south pole of the projection, $\mathbf{v}_{\tau}$. The projection itself is shown on the right. The metric on the sphere is given by

$$
d s^{2}=\frac{4}{\left(1+u^{2}+v^{2}\right)^{2}}\left(d u^{2}+d v^{2}\right)
$$

for coordinates on the plane $u$ and $v$. Over the boundary box in Figure 4 the metric ranges from $4\left(d u^{2}+d v^{2}\right)$ to $3.76\left(d u^{2}+d v^{2}\right)$; there is a maximum distortion of a length in the stereographic projection of $3 \%$.

Though we assume that the trajectory of $\boldsymbol{\alpha}$ is universal, there is no physical constraint on the relation between the quark and lepton sectors. We thus have the freedom to match these two sectors to give the smoothest trajectory. As mentioned previously we also have a freedom in choosing the $U D$ matrix. To find the best matching with experimental data we ranged over, and then applied simplex optimization at good regions in, the parameter space of quark-lepton sector matching matrices and $U D$ matrices. For each point in parameter space tested we found the positions of $\boldsymbol{\alpha}_{t, c, b, s, \tau, \mu}$ and projected them onto the plane. We then fit a cubic line to these points on the plane using a non-linear least squares algorithm. Previous work [5] has shown that the cumulative arc length between $\boldsymbol{\alpha}_{x}$ can be modelled by an exponential function at high scales. Accordingly, we then fit an exponential function to the cumulative arc length, excluding the strange quark and the electron. The strange quark was excluded since the interpretation of its intermediate mass is somewhat uncertain in this scheme. The electron 

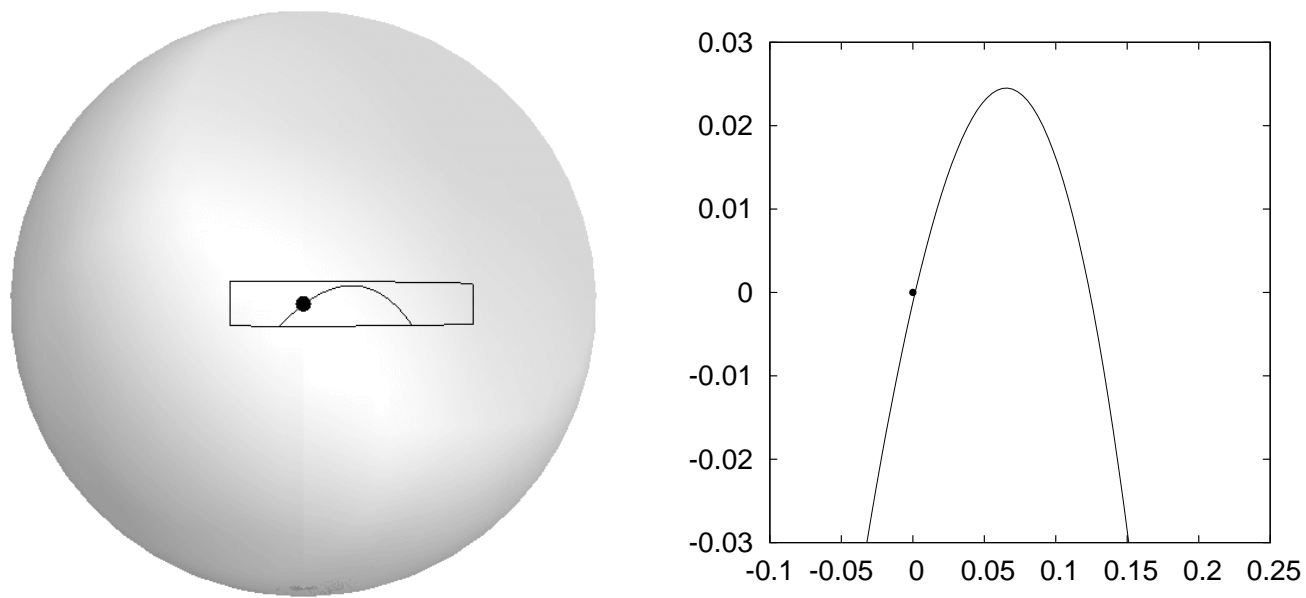

Figure 4: The state vectors and $\boldsymbol{\alpha}$ range over a unit sphere in $\mathbb{R}^{3}$. These are conveniently represented as points on the plane under stereographic projection. The point shown lies at the south pole of the projection. The box on the sphere is the boundary of the plot on the right, and the curve shows the best fit line found.

was excluded firstly since later we will only be interested in the relationship at higher scales and secondly since we do not have good restrictions on the cumulative arc length, as $\boldsymbol{\alpha}_{e}$ can only be constrained to lie on a line. Finally we use the Jarlskog invariant to fix the value of $\theta$ and so determine the magnitudes of the elements of the CKM matrix.

Figure 5 shows the cubic best fit line along with the positions of $\boldsymbol{\alpha}_{x}$ found in [21]. The experimental errors in the masses of the quarks lead to an uncertainty in the position of $\boldsymbol{\alpha}_{c}$ and $\boldsymbol{\alpha}_{s}$. The $1 \sigma$ errors in the quark masses restrict $\boldsymbol{\alpha}_{c}$ and $\boldsymbol{\alpha}_{s}$ to lie on the lines shown. The cubic best fit line is

$$
0.75 u-4.83 u^{2}-9.19 u^{3} .
$$

The cumulative arc length between $\boldsymbol{\alpha}_{x}$ 's was found after mapping the cubic best fit line onto the sphere through an inverse stereographic projection.

Figure [6] shows that the arc lengths between $\boldsymbol{\alpha}_{t}, \boldsymbol{\alpha}_{b}, \boldsymbol{\alpha}_{\tau}, \boldsymbol{\alpha}_{c}$ and $\boldsymbol{\alpha}_{\mu}$ are well fitted by the exponential curve

$$
0.104 \exp \left(-1.228 \log _{10}(\mu)\right)-0.0061
$$

for $\mu$ in $\mathrm{GeV}$. This is in good agreement with the results from the planar 

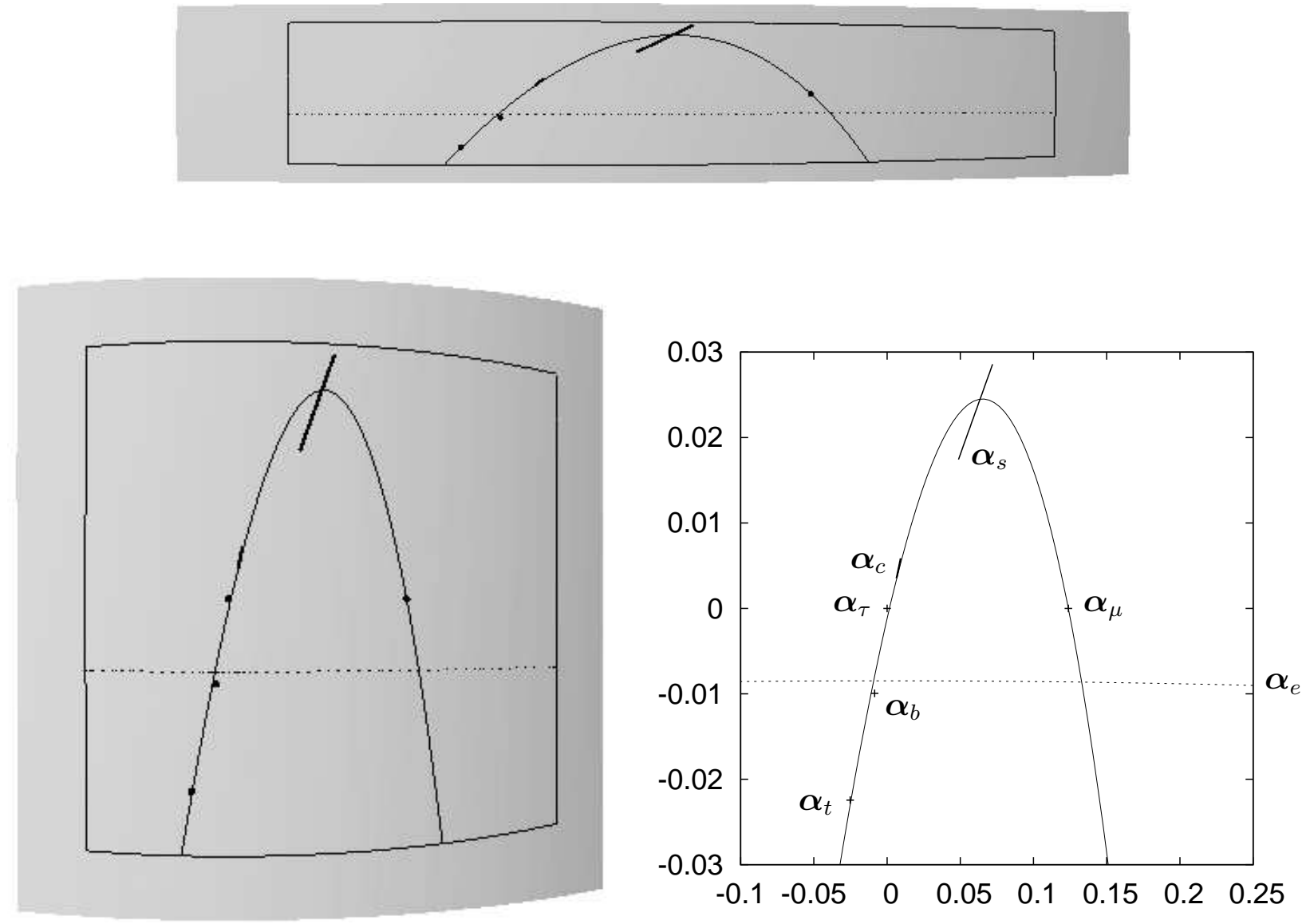

Figure 5: The positions of $\boldsymbol{\alpha}$ at various scales determined partly by experimental constraints and partly by our choices as described in the text. The position of $\boldsymbol{\alpha}_{e}$ is constrained to lie somewhere on the dotted line. The cubic best fit line is shown by the solid line. The top shows these on the sphere, left shows them on an ellipsoid with the axes stretched to match those of the stereographic projection while the right shows the stereographic projection with the positions of $\boldsymbol{\alpha}_{x}$ indicated. 


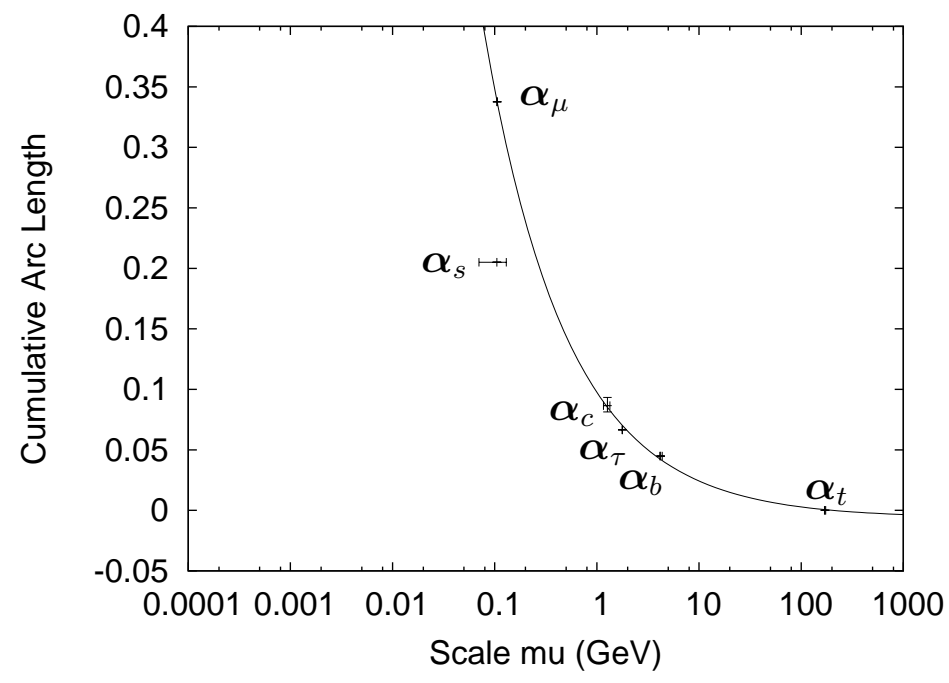

Figure 6: The cumulative arc length along the best fit line measured from $\boldsymbol{\alpha}_{t}$ is well approximated by an exponential curve for all but $\boldsymbol{\alpha}_{s}$. A great circle gives an arc length of $2 \pi$ in these units.

approximation (Figure 3) found in [5] and provides a good fit to data points down to scales of around $100 \mathrm{MeV}$. For the later work on Higgs decay we only need to model the behaviour at high scales.

For this trajectory we find $\omega_{U}=0.09$ radians and $\omega_{D}=0.25$ radians (Figure 7). Fitting a Jarlskog invariant of $J=3.05 \times 10^{-5}$ gives a strong CP angle of 1.45 radians. These results are in line with estimates in [29]. The absolute values of the CKM matrix obtained are:

$$
\left(\begin{array}{ccc}
0.97430 & 0.2252 & 0.00357 \\
0.2251 & 0.97345 & 0.0415 \\
0.00879 & 0.0407 & 0.999134
\end{array}\right)
$$

which can be compared with the experimental values [18]:

$$
\left(\begin{array}{ccc}
0.97419 \pm 0.00022 & 0.2257 \pm 0.0010 & 0.00359 \pm 0.00016 \\
0.2256 \pm 0.0010 & 0.97334 \pm 0.00023 & 0.0415_{-0.0010}^{+0.0010} \\
0.00874_{-0.00037}^{+0.00026} & 0.0407 \pm 0.0010 & 0.999133_{-0.000043}^{+0.000044}
\end{array}\right) .
$$



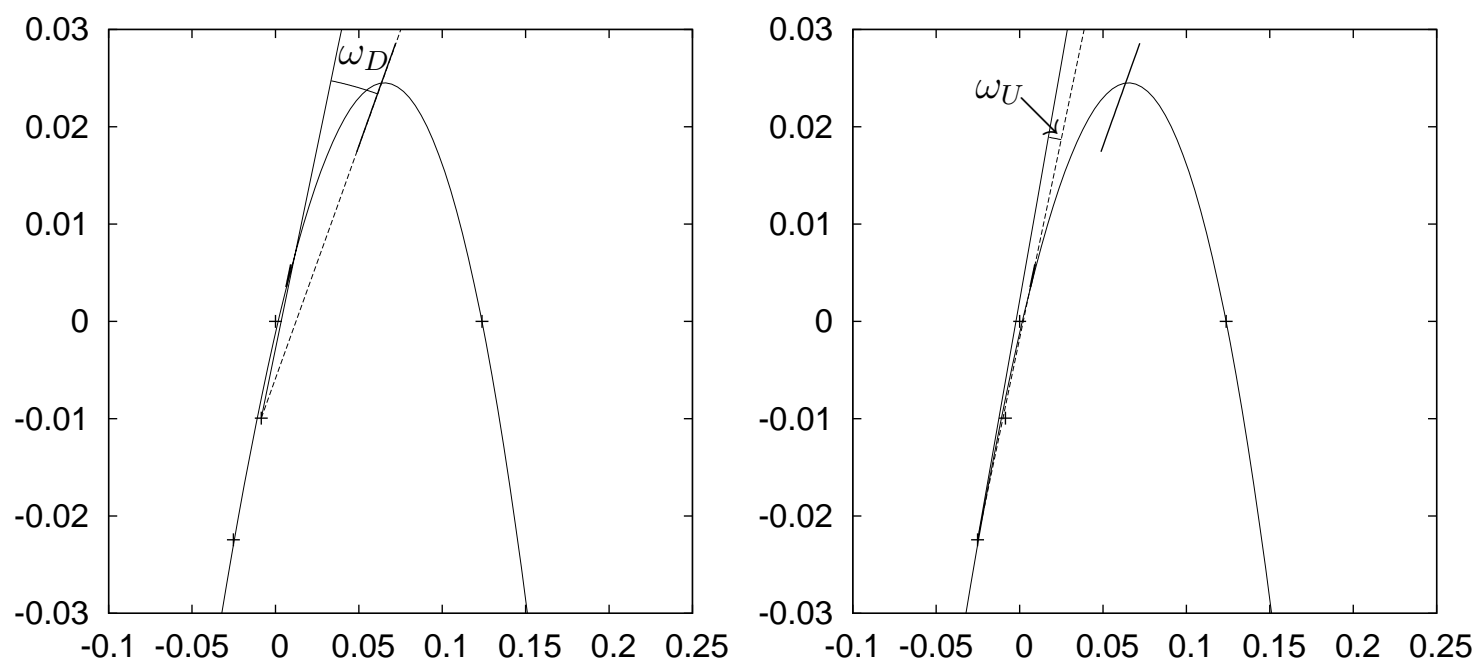

Figure 7: An illustration of the angle $\omega_{D}\left(\omega_{U}\right)$. Here the state vector triad and the Darboux triad have their origins at $\boldsymbol{\alpha}_{b}\left(\boldsymbol{\alpha}_{t}\right)$. The solid line shows $\boldsymbol{\tau}(\mu) \| \dot{\boldsymbol{\alpha}}(\mu)$ and the dashed line shows $\mathbf{v}_{s}\left(\mathbf{v}_{c}\right)$. It should be remembered that the axes are not of equal scale and that this is a stereographic projection of the vectors so the angles cannot be directly read off. 
We find the unitarity angles, defined and measured [18] as

$$
\begin{aligned}
& \alpha=\arg \left(-\frac{V_{t d} V_{t b}^{*}}{V_{u d} V_{u b}^{*}}\right)=\left(88_{-5}^{+6}\right)^{\circ} \\
& \beta=\arg \left(-\frac{V_{c d} V_{c b}^{*}}{V_{t d} V_{t b}^{*}}\right)=\frac{1}{2} \sin ^{-1}(0.681 \pm 0.025), \\
& \gamma=\arg \left(-\frac{V_{u d} V_{u b}^{*}}{V_{c d} V_{c b}^{*}}\right)=\left(77_{-32}^{+30}\right)^{\circ},
\end{aligned}
$$

to be $\alpha=88^{\circ}, \sin (2 \beta)=0.691$ and $\gamma=70^{\circ}$.

From the above analysis [21, one concludes that the recent data cited can all be accommodated, to within the impressive accuracy achieved in experiment and in detail, by a smooth trajectory for $\boldsymbol{\alpha}$ as required by R2M2. It can be noted there that the CP-violating phase, introduced in the manner peculiar to the R2M2 framework as detailed in the preceding subsection, plays a role in achieving this result. Further, as a by-product, the fitted trajectory will be of use to phenomenological applications of the hypothesis, an example of which will follow in the next subsection.

\subsection{The prospect of a charmless Higgs}

In $\S 2.1$, use is made of a special feature of R2M2, namely that of maintaining chiral invariance while leaving all quarks massive, to offer a solution to the strong CP problem which, lying as it does outside the original remit of R2M2, lends strong support to its validity. This feature is however but a consequence of rotation. Can rotation itself then not give direct consequences which can be tested against experiment? At first sight, the answer would seem to be simple. The rotation of the mass matrix implies that what is a mass eigenstate at one scale would no longer appear as one at another scale but as some linear combination of mass eigenstates. The effects would seem thus to lead to flavour-violations and be easily identifiable. Indeed some early efforts have been made [32] to try to explore some of them. These, however, involve an assumption which R2M2 itself cannot justify and which under closer scrutiny cannot easily be maintained. The rotation of the mass matrix, like the running of other quantities, is supposed to be a consequence of renormalization, presupposing therefore an underlying renormalizable theory. Even though one can treat this rotation, as one has done in this paper, as a hypothesis, without specifying the theory on which it is based, there is a tacit limit to its application. The underlying theory, if one knows what 
it is, will give rise, presumably, to other renormalization effects besides the rotation of the mass matrix, and if the investigation one is pursuing involves those other effects, they may counteract or otherwise modify the effects deduced from the rotating mass matrix alone, making them thereby erroneous. Indeed, it was shown [33], using an early phenomenological model [13], that some effects on flavour violations deduced from the rotation of the mass matrix alone would be cancelled automatically by an opposite rotation effect due to wave function renormalization. The mass spectrum and mixing matrices evaluated above with R2M2 alone just happen to be particularly simple "static" single-particle properties which, we believe, are not affected by other effects of renormalization. For anything beyond, however, one has to be wary in making predictions with R2M2 alone, for which only a calculation with a full-blown theory of all renormalization effects can really be a fool-proof guarantee.

Defying this caution, however, let us consider in the R2M2 context the example of Higgs boson decay into fermion-antifermion pairs. This being still, in a sense, a static property, it may perhaps survive better an unsophisticated treatment, and being also timely, in view of the LHC's imminent coming of age, it may perhaps be worth the risk. At least, one has seen no evidence yet of the complications that one has seen before in [33].

To investigate Higgs decay into fermion-antifermion pairs, we will need the Yukawa coupling. One possibility which will give the required factorizable mass matrix (2) is the following:

$$
\mathcal{A}_{Y K}=\rho_{T} \bar{\psi} \boldsymbol{\alpha} \phi_{W} \boldsymbol{\alpha}^{\dagger} \psi
$$

Expanding $\phi_{W}$ about its minimum value $\zeta_{W}$, thus: $\phi=\zeta_{W}+H$, we obtain to zeroth order the fermion mass matrix as in (2) with $m_{T}=\rho_{T} \zeta_{W}$, and to first order the coupling matrix of the Higgs boson to the fermions as:

$$
\Gamma=\rho_{T} \boldsymbol{\alpha} \boldsymbol{\alpha}^{\dagger} .
$$

Superficially, this result looks familiar, namely that the fermion mass and Higgs coupling are proportional to each other. However, as usually meant, the proportionality is between the mass and the Higgs coupling of each of the fermions individually, namely that $m_{i}=\zeta_{W} y_{i}$, with $i$ denoting the individual fermion state, but here it is a proportionality between matrices: $m=\zeta_{W} \Gamma$. And both these matrices rotate. Having seen above that rotation of the mass matrix $m$ alone already leads to intriguing consequences, we shall not be surprised that here too rotation of the Higgs coupling matrix $\Gamma$, which can be thought of as the "Higgs state tensor", will give new interesting results. 
Let us concentrate first on the mode into $c \bar{c}$ pair which gives the most dramatic effect. As usual, for the decay amplitude, one would take the element of the matrix $\Gamma$ in (69) between $c$ state vectors, i.e.

$$
A(H \rightarrow c \bar{c})=\rho_{U}|\langle\mathbf{c} \mid \boldsymbol{\alpha}\rangle|^{2} .
$$

However, since $\boldsymbol{\alpha}$ in (70) now depends on $\mu$, we have to specify at which scale to evaluate it. The usual convention is that, for Higgs decay, quantities which depend on scale should be evaluated at the scale of the Higgs mass, i.e. $\mu=M_{H}$, hence:

$$
A(H \rightarrow c \bar{c})=\rho_{U} \mid\left\langle\mathbf{c}\left|\boldsymbol{\alpha}\left(\mu=M_{H}\right\rangle\right|^{2} .\right.
$$

The Higgs mass is, of course, still unknown, but is limited by recent experiment to be above $115 \mathrm{GeV}$. In other words, it is getting to be near the scale of the top mass $m_{t} \sim 170 \mathrm{GeV}$. In any case, according to Figures 3 and 6 , for example, $\boldsymbol{\alpha}$ is fast approaching its asymptotic value at $\mu=\infty$ and not changing much there already, so that effectively we have $\boldsymbol{\alpha}\left(\mu=M_{H}\right) \sim \boldsymbol{\alpha}\left(\mu=m_{t}\right)$. Recall now, however, that $\boldsymbol{\alpha}\left(\mu=m_{t}\right)$ is by definition $\mathbf{t}$, the state vector for $t$, and orthogonal to $\mathbf{c}$, thus giving a near zero value for (71), the decay amplitude. One concludes therefore that the decay width for the mode $H \rightarrow c \bar{c}$ evaluated with R2M2 in this way will be much suppressed compared with the value expected in the standard model $\sim \rho_{U}^{2} m_{c}^{2}$ which would be the value obtained from (70) if the scale-dependent $\boldsymbol{\alpha}$ were evaluated at $\mu=m_{c}$, not at $\mu=M_{H}$ as one thought it should be. And quite clearly, the suppression is a direct consequence of rotation.

Similar arguments obviously apply to the other fermions of the second heaviest generation $s$ and $\mu$, although the suppression will be less drastic in both these cases, since $\mathbf{b}=\boldsymbol{\alpha}\left(\mu=m_{b}\right)$ and $\boldsymbol{\tau}=\boldsymbol{\alpha}\left(\mu=m_{\tau}\right)$ are neither of them as close to $\boldsymbol{\alpha}\left(\mu=M_{H}\right)$ as $\mathbf{t}=\boldsymbol{\alpha}\left(\mu=m_{t}\right)$ is. On the other hand, we notice that the decay into the heaviest generation fermions of any type are not much affected by rotation, these being dependent on the cosines of the rotation angles involved, not on the sines of the angles as the second generation fermions are. This means that the branching ratios into the second heaviest to the heaviest generations will all be much suppressed.

It is not difficult to give some actual estimates for the suppression factors since from fitting the mass and mixing data before, we have already a fair idea of how $\boldsymbol{\alpha}$ behaves as a function of $\mu$. For example, reading from Figure 3] or 6 the angle corresponding to $\boldsymbol{\alpha}\left(\mu=M_{H}\right)$, which is straightforward (apart from a minor technical point explained in 34] concerning a possible 

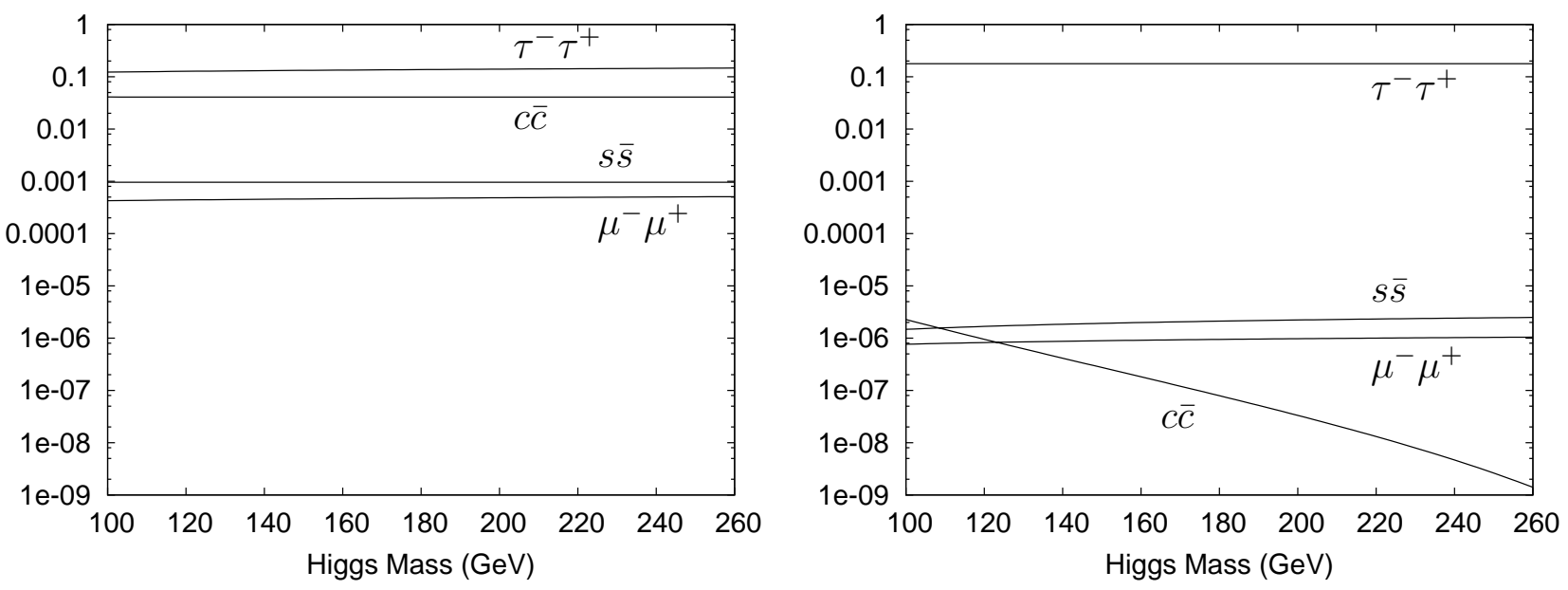

Figure 8: $\Gamma(H \rightarrow x \bar{x}) / \Gamma(H \rightarrow b \bar{b})$ for various final state particles as predicted by the standard model (left) and the rotating mass matrix hypothesis (right).

shift in calibration of scales which makes, however, little difference to the conclusions) one has for $M_{H}=150 \mathrm{GeV}$ :

$$
\frac{\Gamma(H \rightarrow c \bar{c})}{\Gamma(H \rightarrow b \bar{b})} \sim 4.3 \times 10^{-7} .
$$

which is nearly 5 orders of magnitude less than the standard model expectation of $\sim m_{c}^{2} / m_{b}^{2} \sim 0.09$. Similar estimates for the corresponding branching ratios for $s$ and $\mu$ are, again for $M_{H}=150 \mathrm{GeV}$, respectively $1.8 \times 10^{-6}$ and $2.9 \times 10^{-6}$ as compared to standard model expectations of about $6 \times 10^{-4}$ for both.

Making use of the more detailed 3-G fit to mass and mixing data described in $\S 2.2$, one will be able to derive the branching ratios of anomalously suppressed modes involving the lightest generations too, although not as transparently. Some such branching ratios are shown in Figure 8 on the right, with comparison with standard model results on the left [35. The lightest generations had branching ratios less than $10^{-9} \times \Gamma(H \rightarrow b \bar{b})$.

Apart from the modes considered above which are anomalously suppressed, there is another category of anomalous modes with a similar origin. The Yukawa coupling in (171) for a general $\mu$ has usually nondiagonal matrix elements between state vectors of fermions of the same type but from different generations, leading to flavour-violating decays of the sort: $H \rightarrow \tau \bar{\mu}$. 

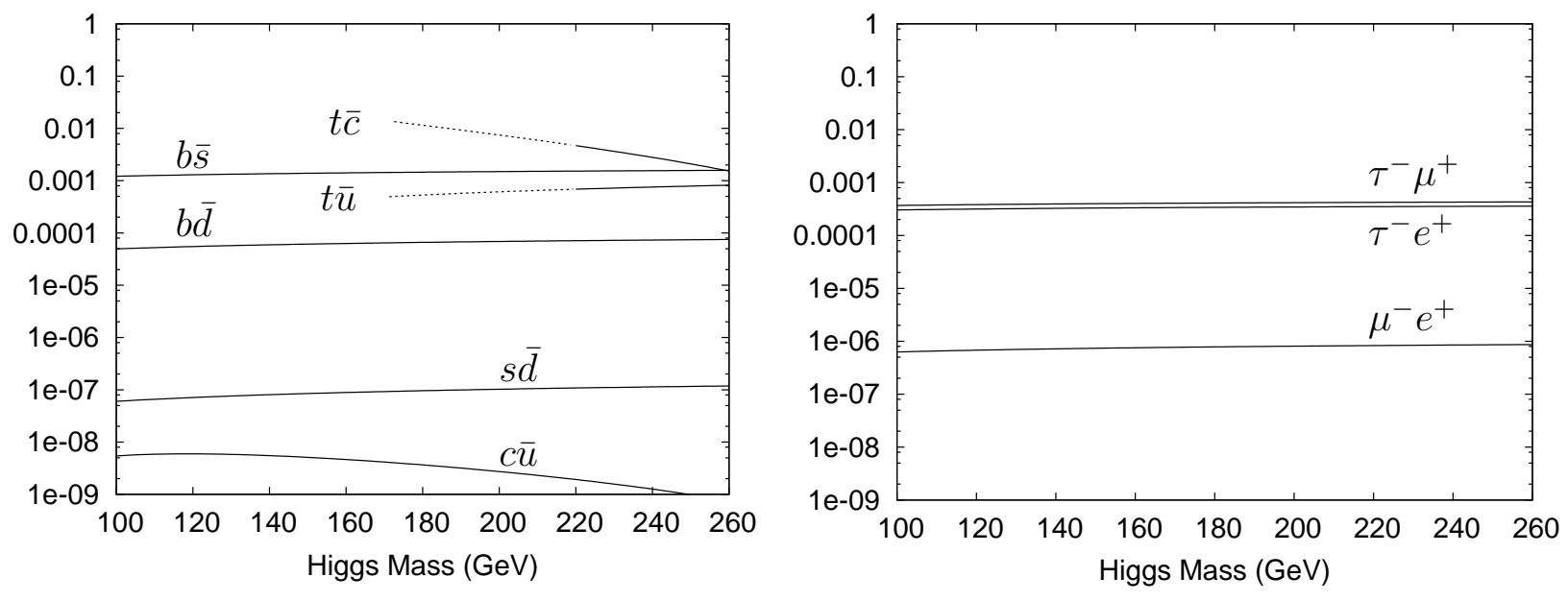

Figure 9: $\Gamma(H \rightarrow x \bar{y}) / \Gamma(H \rightarrow b \bar{b})$ for various flavour violating decays as predicted by the rotating mass matrix hypothesis. Note that $\Gamma(H \rightarrow y \bar{x})=$ $\Gamma(H \rightarrow x \bar{y})$. Below around $220 \mathrm{GeV}$, indicated by the dotted lines, threshold effects will influence the $t \bar{c}$ and $t \bar{u}$ decay modes.

Branching ratios predicted from Figure 3 or the fit in $\S 2.2$ for such modes are generally quite small, for example, from the former, one has, again for $M_{H}=150 \mathrm{GeV}$ :

$$
\frac{\Gamma(H \rightarrow \tau \bar{\mu})}{\Gamma(H \rightarrow b \bar{b})}=\frac{m_{\tau}^{2}}{m_{b}^{2}} \frac{\cos ^{2} \theta_{H \tau} \sin ^{2} \theta_{H \tau}}{\cos ^{4} \theta_{H b}} \sim 7.7 \times 10^{-4}
$$

which, being so distinctive though small, may eventually be seen. The more general conclusions from the fit in [21] is summarized in Figure 9.

Predictions on flavour-violation are often troublesome in that the effect may propagate and lead to violations in other areas already very strongly bounded by experiment. Of most danger in this case that one can identify is the implication of Higgs exchange for the mass difference between the two neutral-strange $B$ mesons, for which one obtains from Figure 3 the following:

$$
m_{B_{s H}^{0}}-m_{B_{s L}^{0}} \sim 4.8 \times 10^{-10} \mathrm{MeV}
$$

which is more than an order of magnitude below the experimentally measured value of $117 \times 10^{-10} \mathrm{MeV}$. Given the difficulty of making accurate theoretical predictions for such hadronic quantities, the Higgs contribution 
is unlikely to be noticeable at present and thus causes no problem, but it may be something to look for in future when experimental measurement and theoretical interpretation both continue to improve. As for other implied flavour-violations like $\tau \rightarrow \mu \mu \bar{\mu}$ decay, the estimates are so low as to cause no worry for a long time to come. Thus, the conclusion for the moment is that the prediction of flavour-violation in Higgs decay, such as (73) above, does not lead to contradiction to experiment elsewhere.

For more details and more examples of similar results, the reader is referred to the original research papers [34, 21].

These two "predictions", namely of the anomalous suppression of modes like $c \bar{c}$ and of flavour-violations in modes like $\tau \bar{\mu}$, are exciting in that they are exotic and can in principle be checked soon against experiment at the LHC, although, we are told, some channels may initially be difficult to identify. However, they are by no means as solid as the result in $\S 2.1$, depending as they do on further assumptions the validity of which one is in no position at present to assess. Indeed, as far as these "predictions" are concerned, R2M2 seems to be placed in a, perhaps fortunate but certainly undignified, "winwin" situation. If these "predictions" were to be confirmed by experiment, then R2M2 may claim success since no other scheme is known to give such predictions. On the other hand, if they were to be disproved by experiment, one can always lay the blame on the additional assumptions which have been thrown in to derive the results and learn instead from the failure. Only time and further work, however, will be able to clarify the situation.

\section{Summary and Remarks}

The R2M2 hypothesis has two components: (a) that the fermion mass matrix is of rank one, (b) that this matrix rotates. The first is old, having been suggested some 30 years ago; what is relatively new is the second, namely that the mass matrix changes orientation in generation space with changing scale. And this simple addition is seen immediately to lead to (i) a hierarchical mass spectrum and (ii) mixing matrices for fermions with the qualitative features observed in experiment. A closer examination confirms that the hypothesis is fully consistent with current data, in detail and to within the impressively small experimental errors recently achieved. Exceptions, such as the masses of the light quarks $u$ and $d$, are seen not so much as inconsistencies than as features which the hypothesis have so far failed to explain due to the common lack of understanding at present of colour confinement, inhibiting 
thereby direct comparison of the hypothesis to experiment.

An unusual consequence of the R2M2 hypothesis is that chiral symmetry can be maintained in the action while still allowing all fermions to have nonzero though hierarchical masses. Apart from repercussions probably elsewhere, it is shown in particular to offer (iii) a novel solution to the strong $\mathrm{CP}$ problem by linking it via rotation to the $\mathrm{CP}$-violating phase in the CKM matrix, even giving (iv) a Jarlskog invariant in the CKM matrix of the order $3 \times 10^{-5}$ experimentally observed, for a theta-angle of order unity in the QCD action. This is a particularly gratifying result in that it is obtained in an entirely different domain of physics than that for which the R2M2 hypothesis was originally intended, and could thus be regarded as a nontrivial check on the hypothesis. The result is also of some theoretical significance in that it has linked together the theta-angle of topological origin in QCD to the KM phase in the CKM matrix belonging to the weak current, two concepts previously thought to be entirely separate.

Further, R2M2 is seen to predict (v) anomalies in Higgs decay which should be testable at LHC in the near future, although these depend on further assumptions.

Taken together, the results (i) - (v) seem a fair recommendation for R2M2 to be considered as a means to an end by model builders who are looking behind the standard model with the aim of explaining its many idiosyncrasies. Any model, it seems, which can produce a rank-one fermion mass matrix rotating with changing scale in generation space at sufficient speed, would have a fair chance of success. For this reason, although we ourselves have suggested one such model with R2M2 as a consequence [15, 14, 11], in the present review R2M2 is cast deliberately as a phenomenological hypothesis and only those results derivable from R2M2 are discussed which are independent of how the rotation is generated. This will make it more useful, we hope, as a guide for fellow model builders starting from different premises with completely different ideas from ours.

\section{References}

[1] Steven Weinberg, Phys. Rev. D7 (1973) 2887.

[2] H. Fritsch, Nucl. Phys. B 155, 189 (1978).

[3] H. Harari, H. Haut, and J. Weyers, Phys. Lett. B 78, 459 (1978) 
[4] See e.g. J.A.M Vermaseren, S.A Larin and T van Ritbergen, Phys. Lett. B405 (1997) 327.

[5] José Bordes, Chan Hong-Mo and Tsou Sheung Tsun, Eur. Phys. J. C 27 (2003) 189-200, hep-ph/0203124.

[6] N. Cabibbo, Phys. Rev. Lett. 10, 531 (1963).

[7] M. Kobayashi and K. Maskawa, Prog. Theor. Phys. 49, 282 (1972).

[8] H. Arason, D.J. Castaño, B. Kesthelyi, S. Mikaelian, E.J. Piard, P. Ramond, and B.D. Wright, Phys. Rev. D46, 3945, (1992).

[9] See e.g. B. Grzadkowski, M. Lindner and S. Theisen, Phys. Lett. B198, 64 (1987).

[10] B. Pontecorvo, Zh. Eksp. Teor. Fiz. 33, 549 (1957) and 34, 247 (1958); Z. Maki, M. Nakagawa and S. Sakata, Progr. Theor. Phys. 28, 870 (1962).

[11] Chan Hong-Mo and Tsou Sheung Tsun, Eur. Phys. J. C52, 635-663 (2007); arXiv:hep-ph/0611364.

[12] José Bordes, Chan Hong-Mo and Tsou Sheung Tsun, Eur. Phys. J. C. 10, 63 (1999), hep-ph/9901440.

[13] José Bordes, Chan Hong-Mo, Jacqueline Faridani, Jakov Pfaudler, and Tsou Sheung Tsun, Phys. Rev. D58, 013004, (1998), hep-ph/9712276.

[14] Michael J Baker, José Bordes, Chan Hong-Mo and Tsou Sheung Tsun, in preparation.

[15] Chan Hong-Mo and Tsou Sheung Tsun, in preparation.

[16] EJ Le Couteur, Proc. Roy. Soc. (London) A256 (1960) 115; RG Newton, J. Math. Phys. 2 (1961) 188.

[17] See e.g. L.P. Eisenhart, A Treatise on the Differential Geometry of Curves and Surfaces, Ginn and Company 1909, Boston; M.P. do Carmo, Differential Geometry of Curves and Surfaces, Prentice-Hall 1976, Englewood Cliffs, New Jersey.

[18] C. Amsler et al. (Particle Data Group), Phys. Lett. B667, 1 (2008) and 2009 partial update for the 2010 edition; http://hepdata.cedar.ac.uk/lbl/ 
[19] C. Caso et al. (PDG), The European Physical Journal C3, 1, (1998).

[20] JS Palmer and Tsou Sheung Tsun, Mod. Phys. Lett. A18 (2003) 807-815; hep-ph/0304013.

[21] Michael J Baker and Tsou Sheung Tsun, Eur. Phys. J. C70 (2010) 1009; DOI 10.1140/epjc/s100052-010-1506-0, arXiv:1005.2676 [hep-ph].

[22] See for example: S. Weinberg, The Quantum Theory of Fields II (Cambridge University Press, New York, 1996).

[23] C.A. Baker et al., Phys. Rev. Lett. 97131801 (2006).

[24] R.D. Peccei and H. Quinn, Phys. Rev. Lett. 38, 1440 (1977); Phys. Rev. D 16, 1791 (1977).

[25] S. Weinberg, Phys. Rev. Lett. 40, 223 (1978).

[26] Jakov Pfaudler, arXiv: hep-th/9703058.

[27] Chan Hong-Mo and Tsou Sheung Tsun, Acta Phys. Polonica, 33 (2002) 4041, hep-ph/0303010.

[28] José Bordes, Chan Hong-Mo and Tsou Sheung Tsun, arXiv:0707.3358, Int. J. Mod. Phys. A 24, 101 (2009).

[29] José Bordes, Chan Hong-Mo and Tsou Sheung Tsun, arXiv:1002.3542 [hep-ph], ] Int. J. Mod. Phys. A25 (2010) 5897-5911.

[30] C. Jarlskog, Z. Phys. C 29491 (1985); Phys. Rev. Lett. 55, 1039 (1985).

[31] José Bordes, Chan Hong-Mo, Jakov Pfaudler, and Tsou Sheung Tsun, Phys. Rev. D58, 053006, (1998), hep-ph/9802436.

[32] José Bordes, Chan Hong-Mo and Tsou Sheung Tsun, Phys. Rev. D63, 016006 (2001), hep-ph/0006338.

[33] José Bordes, Chan Hong-Mo and Tsou Sheung Tsun, Phys. Rev. D65, 093006, (2002), hep-ph/0111369.

[34] José Bordes, Chan Hong-Mo and Tsou Sheung Tsun, Eur. Phys. J. C65 (2010) 537-542; arXiv:0908.1750 [hep-ph].

[35] M. Spira, HDECAY version 3.51 (July 2009). Available: http://people.web.psi.ch/spira/proglist.html. 